\title{
Reduction of quantum systems on Riemannian manifolds with symmetry and application to molecular mechanics
}

\author{
AUTHOR(S): \\ Tanimura, S; Iwai, T
}

\section{CITATION:}

Tanimura, S ... [et al]. Reduction of quantum systems on Riemannian manifolds with symmetry and application to molecular mechanics. JOURNAL OF MATHEMATICAL PHYSICS 2000, 41(4): 1814-1842

\section{ISSUE DATE:}

2000-04

URL:

http://hdl.handle.net/2433/50320

\section{RIGHT:}

Copyright 2000 American Institute of Physics. This article may be downloaded for personal use only. Any other use requires prior permission of the author and the American Institute of Physics. 


\title{
Reduction of quantum systems on Riemannian manifolds with symmetry and application to molecular mechanics
}

\author{
Shogo Tanimura ${ }^{a}$ \\ Department of Engineering Physics and Mechanics, Kyoto University, \\ Kyoto 606-8501, Japan \\ Toshihiro Iwaib) \\ Department of Applied Mathematics and Physics, Kyoto University, \\ Kyoto 606-8501, Japan
}

(Received 2 July 1999; accepted for publication 19 November 1999)

This paper deals with a general method for the reduction of quantum systems with symmetry. For a Riemannian manifold $M$ admitting a compact Lie group $G$ as an isometry group, the quotient space $Q=M / G$ is not a smooth manifold in general but stratified into a collection of smooth manifolds of various dimensions. If the action of the compact group $G$ is free, $M$ is made into a principal fiber bundle with structure group $G$. In this case, reduced quantum systems are set up as quantum systems on the associated vector bundles over $Q=M / G$. This idea of reduction fails, if the action of $G$ on $M$ is not free. However, the Peter-Weyl theorem works well for reducing quantum systems on $M$. When applied to the space of wave functions on $M$, the Peter-Weyl theorem provides the decomposition of the space of wave functions into spaces of equivariant functions on $M$, which are interpreted as Hilbert spaces for reduced quantum systems on $Q$. The concept of connection on a principal fiber bundle is generalized to be defined well on the stratified manifold $M$. Then the reduced Laplacian is well defined as a self-adjoint operator with the boundary conditions on singular sets of lower dimensions. Application to quantum molecular mechanics is also discussed in detail. In fact, the reduction of quantum systems studied in this paper stems from molecular mechanics. If one wishes to consider the molecule which is allowed to lie in a line when it is in motion, the reduction method presented in this paper works well. (C) 2000 American Institute of Physics. [S0022-2488(00)01104-X]

\section{INTRODUCTION}

Symmetry has always played an important role in mechanics. When a Hamiltonian system admits a symmetry group, the system reduces to a Hamiltonian system of less degrees of freedom. Reduction of Hamiltonian systems with symmetry is established by Marsden and Weinstein ${ }^{1}$ and is explained in a textbook. ${ }^{2}$ The reduction method has found a wide variety of applications. In particular, the notion of moment map, which is a key word in the reduction method, has been introduced in differential geometry.

The reduction method is applicable to molecular mechanics, both classical and quantum. In fact, the translational invariance allows one to separate the relative motion of atoms from the motion of the center of mass. Contrary to this, the rotational invariance of molecules is not able to separate the vibrational motion from the rotational motion of the whole molecular system, which was shown by Guichardet. ${ }^{3}$ The nonseparability of vibration and rotation of a molecule is an underlying principle that allows cats to fall on their legs when launched in the air. While they have

\footnotetext{
${ }^{a)}$ Electronic mail: stani@yukawa.kyoto-u.ac.jp

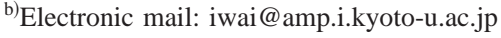


zero angular momentum and is free from external torque during the fall, they can make a rotation after a vibrational motion. It was Guichardet ${ }^{3}$ who gave a decisive answer to this seemingly strange fact. He defined a natural connection on the center-of-mass system and thereby applied the holonomy theorem to show that the existence of nonvanishing curvature results in the nonseparability of vibration and rotation. It is to be noted here that the center-of-mass system is made into a fiber bundle if collinear configurations of the molecule are gotten rid of. The connection is defined on this restricted center-of-mass system.

In spite of the nonseparability of vibration and rotation, the reduction method is still applicable. One of the authors (T.I.) applied the reduction method of Marsden and Weinstein to the molecular system by the use of conservation of angular momentum and gave a reduced Hamiltonian system, ${ }^{4}$ according to which the "internal" motion of molecules is coupled with rotation through a kind of gauge field. The expression of the reduced equation of motion in terms of local coordinates is given in Ref. 5. He also constructed the Schrödinger equation to describe the internal motion of molecules in both two and three dimensions, using differential geometry of fiber bundles and connections. ${ }^{6-8}$ However, in the previous papers, ${ }^{6-8}$ collinear configurations of the molecule in which all the atoms are aligned in a line in $\boldsymbol{R}^{3}$ were out of consideration for the reason that the collinear configurations form a singular point set which prevents the center-of-mass system from being made into a principal fiber bundle. The bundle picture is extended to be applicable to a system of rigid bodies. Classical and quantum mechanics for the system of jointed identical axially symmetric cylinders is treated in Ref. 9.

It is Kummer ${ }^{10}$ who first discussed the reduction of the cotangent bundle of a principal fiber bundle with a connection. He may have been aware of a use of the connection in many-body systems. However, he did not refer to the center-of-mass system as a principal fiber bundle in general, but referred to a planar three-body system as an $S^{1}$-bundle. He claimed also that the reduction method had been applied to the planar three-body system by Satzer. ${ }^{11}$ Guichardet is the first who showed that the connection defined on the restricted center-of-mass system as a principal fiber bundle played an important role in the study of many body systems; he showed that the holonomy group of the connection coincides with the structure group by the use of the AmbroseSinger holonomy theorem, along with the conclusion that any rotation can be realized as a holonomy associated with a closed loop in the base manifold (or a shape space).

This paper has an aim to improve the previous theory so that it may be applicable on the whole center-of-mass system which includes the singular point set stated above. Since the centerof-mass system is endowed with a natural Riemannian metric and admits the action of $\mathrm{SO}(3)$ as an isometry group, a general setting to start with is that a configuration space $M$ is a Riemannian manifold on which a compact Lie group $G$ acts by isometry. For a quantum system on $M$ with symmetry Lie group $G$, the reduced quantum system by symmetry is to be defined on the quotient space $Q=M / G$. However, a problem arises since the $Q$ is not a smooth manifold in general, which may include singular points. A part of $Q$, denoted by $Q_{\mu}$, is a smooth manifold, which is called the internal or shape space endowed with local coordinates describing the internal degrees of freedom of the molecule. One can set up a reduced quantum system on the smooth manifold $Q_{\mu} \cdot{ }^{6,7}$ However, if one considers the whole $Q$, then a question arises as to how wave functions of internal coordinates should behave on the singular point set. This article will provide a general formulation to describe quantum mechanics of a reduced system with singular points taken into account.

Let $L_{2}(M)$ be the Hilbert space of square integrable functions on the Riemannian manifold $M$, which is to be looked upon as the space of wave functions on the center-of-mass system. The group action $G \times M \rightarrow M ;(g, x) \mapsto g x$ induces a unitary representation of $G$ in $L_{2}(M)$ through the action $G \times L_{2}(M) \rightarrow L_{2}(M) ;(g, f(x)) \mapsto f\left(g^{-1} x\right)$. This representation will be decomposed into irreducible ones to give rise to representation subspaces of $L_{2}(M)$ accordingly. To get an idea of the decomposition of $L_{2}(M)$, the Peter-Weyl theorem on unitary representations of compact groups is of great help, since the theorem is understood to provide the decomposition of the Hilbert spaces of functions on groups. The decomposition of $L_{2}(M)$ is then performed in a similar manner, to define the spaces of equivariant functions on $M$ according to respective irreducible 
unitary representations of $G$. These spaces will give rise to wave functions reduced by the use of the angular momentum conservation, since choosing an irreducible unitary representation of the isometry group amounts to keeping an angular momentum eigenvalue fixed. The equivariant functions will be shown to satisfy a good boundary condition, in a natural manner, on the set of singular points. The general formalism for reducing wave functions is applicable to molecular mechanics. In particular, a triatomic molecule will be studied in detail.

The idea of using the Peter-Weyl theorem for quantization is old; it dates back to Casimir's work on quantum mechanics of rigid body in 1931. The idea of using the invariant functions to set up a reduced quantum system by symmetry is also old; for example, the quantum theories of electromagnetism ${ }^{12}$ or gravitation ${ }^{13,14}$ are described by gauge invariant and reparametrization invariant states, respectively. The idea of using equivariant functions is not new, either; it has already been introduced by T.I. (Refs. 8,7) in molecular quantum mechanics and by Landsman and Linden ${ }^{15}$ in quantization on homogeneous spaces, respectively. A point to make in the present paper is to extend the concept of equivariant functions on a principal fiber bundle to any manifold on which a compact group dose not necessarily act freely.

There are other methods of reduction by symmetry. A more algebraic formulation was set up by Landsman ${ }^{16}$ and Wren, ${ }^{17}$ who used a representation theory of $C^{*}$ algebras due to Rieffel. ${ }^{18}$ The present formulation is rather geometric and comprehensible in applications. On the other hand, the path integral formulation is compatible with a method of reduction by symmetry. It has been shown ${ }^{15,19,20}$ that path integral over a Lie group $G$ is reduced to path integral over a homogeneous space $Q=G / H$ when the system possesses symmetry given by a subgroup $H$. However, this method is only applicable to homogeneous spaces, which are free from singularity, while the method proposed in the present paper will be applicable even when the quotient space $Q$ $=M / G$ is not a smooth manifold, as will be shown later.

On the other hand, Śniatycki and Weinstein ${ }^{21}$ and other people ${ }^{22}$ have studied reduction and quantization of symplectic systems with symmetry. They consider reduction of symplectic manifold when the momentum map takes a singular value and therefore the level set does not form a smooth manifold. In our context, singularity refers to points of a configuration space which admit larger isotropy groups than those at generic points. The subject to be considered here is different from what has been considered in Refs. 22,21, and a relation between these subjects will not be examined.

The plan of the present paper is as follows: In Sec. II, the Peter-Weyl theorem on unitary representations of compact groups is reviewed briefly. This theorem is extended to be applied to a unitary representation of a compact Lie group $G$ in a Hilbert space $\mathcal{H}$ in order to decompose $\mathcal{H}$ into a series of invariant subspaces. The decomposition procedure is then applied to the unitary representation of $G$ in the Hilbert space $L_{2}(M)$. As far as the decomposition is concerned, $M$ is assumed only to be equipped with a $G$-invariant measure, and does not need to be a Riemannian manifold. The above stated decomposition of $L_{2}(M)$ proves to be a decomposition into a series of spaces of "equivariant" functions. Section III contains a study of geometric structure of the $G$-manifold $M$, where the assumption is not yet made that $M$ is a Riemannian manifold. It will be shown that the orbit space $Q=M / G$ becomes not a smooth manifold but a collection of smooth manifolds of various dimensions, which structure is called stratification. With this stratification taken into account, a connection on $M$, equivariant functions on $M$, and covariant derivatives will be defined. In Sec. IV, $M$ is assumed to be a Riemannian manifold, and $G$ to act on $M$ by isometry. The Laplacian acting on smooth functions on $M$ will be reduced to be defined on each space of equivariant functions according to the decomposition of $L_{2}(M)$, which will provide a reduced quantum system on $Q$. The reduced systems are well defined. In fact, the equivalence condition will provide a boundary condition on equivariant functions at singular points. As a simple example, the Laplacian on $\boldsymbol{R}^{2}$ will be studied. In Sec. V, the general formalism developed in the preceding sections is applied to molecular mechanics. A general setting for $\mathrm{N}$-atomic molecules will be established, and then triatomic molecules will be studied in detail. Sec. VI contains conclusions and discussions. 


\section{REDUCTION OF QUANTUM SYSTEMS WITH SYMMETRY}

\section{A. The Peter-Weyl theorem}

We start with a brief review of the Peter-Weyl theorem, which will provide a key idea to the reduction method for quantum dynamical systems with symmetry.

Let $G$ be a compact Lie group. Then there is the unique Haar measure $\mu_{G}$ on $G$ which is normalized to satisfy $\int_{G} d \mu_{G}=1$. Let $L_{2}(G)$ denote the space of all the square integrable functions on $G, L_{2}(G):=\left\{f:\left.G \rightarrow C\left|\int_{G}\right| f(g)\right|^{2} d \mu_{G}(g)<\infty\right\}$, which is equipped with the inner product

$$
\left\langle f, f^{\prime}\right\rangle:=\int_{G} \overline{f(g)} f^{\prime}(g) d \mu_{G}(g)
$$

for $f$ and $f^{\prime}$ of $L_{2}(G)$.

Let $\rho^{\chi}: G \rightarrow U\left(\mathcal{H}^{\chi}\right)$ denote an irreducible unitary representation of $G$ on a Hilbert space $\mathcal{H}^{X}$ of finite dimension $d^{x}:=\operatorname{dim} \mathcal{H}^{x}$, where $\chi$ is to label all of inequivalent irreducible unitary representations of $G$, and $U\left(\mathcal{H}^{\chi}\right)$ denotes the space of unitary operators on $\mathcal{H}^{\chi}$. By $\rho_{i j}^{\chi}(g)$ we denote the matrix elements of $\rho^{\chi}(g)$ with respect to some orthonormal basis of $\mathcal{H}^{X}$, where indices range over $i, j=1,2, \ldots, d^{\chi}$.

The Peter-Weyl theorem then states that all the functions $\left\{\sqrt{d^{\chi}} \rho_{i j}^{\chi}(g)\right\}_{\chi, i, j}$ form a complete orthonormal set (CONS) in $L_{2}(G)$. Namely, one has the orthonormality relations,

$$
d^{\chi} \int_{G} \overline{\rho_{i j}^{\chi}(g)} \rho_{k l}^{\chi^{\prime}}(g) d \mu_{G}(g)=\delta^{\chi \chi^{\prime}} \delta_{i k} \delta_{j l}
$$

along with the completeness condition that if

$$
\int_{G} \overline{\rho_{i j}^{\chi}(g)} f(g) d \mu_{G}(g)=0
$$

for all $\chi, i, j$, then $f \equiv 0$. Hence, any function $f \in L_{2}(G)$ can be expanded in a Fourier series in $\left\{\sqrt{d^{\chi}} \rho_{i j}^{\chi}\right\}_{\chi, i, j}$, so that one has

$$
\begin{aligned}
f(h) & =\sum_{\chi, i, j} d^{\chi} \rho_{i j}^{\chi}(h) \int_{G} \overline{\rho_{i j}^{\chi}(g)} f(g) d \mu_{G}(g)=\sum_{\chi, i} d^{\chi} \int_{G} \rho_{i i}^{\chi}\left(h g^{-1}\right) f(g) d \mu_{G}(g) \\
& =\sum_{\chi, i} d^{\chi} \int_{G} \rho_{i i}^{\chi}(g) f\left(g^{-1} h\right) d \mu_{G}(g) .
\end{aligned}
$$

The expansion formula (2.4) is put formally in a compact form,

$$
\sum_{\chi, i} d^{\chi} \rho_{i i}^{\chi}(g)=\delta(g)
$$

where $\delta(g)$ is Dirac's delta function on $G$ with respect to the measure $\mu_{G}$. The Peter-Weyl theorem also implies that

$$
L_{2}(G) \cong \oplus_{\chi}\left(\left(\mathcal{H}^{\chi}\right)^{*} \otimes \mathcal{H}^{\chi}\right),
$$

where it is to be noted that $\left(\mathcal{H}^{x}\right)^{*} \otimes \mathcal{H}^{x}$ is isomorphic with the direct sum of $d^{x}$ copies of $\mathcal{H}^{x}$.

\section{B. Method of reduction}

We now apply the Peter-Weyl theorem to a quantum dynamical system with symmetry to obtain a series of reduced systems. A quantum dynamical system is defined to be a pair $(\mathcal{H}, H)$ of a Hilbert space $\mathcal{H}$ and a Hamiltonian $H$, where $H$ is a self-adjoint operator on $\mathcal{H}$. Suppose that the 
system $(\mathcal{H}, H)$ admits a compact Lie group $G$ as a symmetry group, namely, each element $g$ $\in G$ is represented as a unitary operator $U(g)$ which acts on $\mathcal{H}$, commuting with $H$.

In view of (2.4), we define, for each label $(\chi, i)$, an operator $P_{i}^{\chi}$ on $\mathcal{H}$ to be

$$
P_{i}^{\chi}:=d^{\chi} \int_{G} \rho_{i i}^{\chi}(g) U(g) d \mu_{G}(g),
$$

which apparently commutes with $H$. From the $G$-invariance of the measure $\mu_{G}$ and from the orthonormality relations (2.2), it follows that

$$
\left(P_{i}^{\chi}\right)^{\dagger}=P_{i}^{\chi}, \quad P_{i}^{\chi} P_{j}^{\chi^{\prime}}=\delta^{\chi \chi^{\prime}} \delta_{i j} P_{i}^{\chi} .
$$

Further, the completeness condition (2.5) implies that

$$
\sum_{\chi, i} P_{i}^{\chi}=\mathrm{id}_{\mathcal{H}}
$$

where $\operatorname{id}_{\mathcal{H}}$ is the identity operator on $\mathcal{H}$. Equations (2.8) and (2.9) show that the set $\left\{P_{i}^{\chi}\right\}_{\chi, i}$ forms a family of orthogonal projection operators on $\mathcal{H}$, bringing about the orthogonal decomposition of $\mathcal{H}$,

$$
\mathcal{H}=\oplus_{\chi, i} \operatorname{Im} P_{i}^{\chi}
$$

which is an analog to (2.6). Moreover, each subspace $\operatorname{Im} P_{i}^{\chi}$ is invariant under the action of the Hamiltonian $H$. Thus the dynamical system $(\mathcal{H}, H)$ is broken up into a family of subsystems $\left(\operatorname{Im} P_{i}^{X}, H\right)$ labeled by $(\chi, i)$. We call each system $\left(\operatorname{Im} P_{i}^{X}, H\right)$ a reduced quantum dynamical system.

In the language of physics, the pair $(\chi, i)$ labels conserved quantities associated with the symmetry group $G$, and thereby define a closed dynamical system that consists of the states with the assigned conserved quantities. For example, if the original system has $G=\mathrm{SU}(2)$ symmetry, the angular momentum is conserved. The states labeled by $(j, m)$ have the total angular momentum and the component of the angular momentum fixed at $J^{2}=j(j+1)$ and $J_{3}=m$, respectively, and are described as vector-valued functions with $2 j+1$ components.

\section{Characterization of the reduced system}

To gain a deeper insight into the decomposition (2.10), we introduce an operator on $\mathcal{H}$ by

$$
V_{i j}^{\chi}:=d^{\chi} \int_{G} \rho_{i j}^{\chi}(g) U(g) d \mu_{G}(g)
$$

for each label $\chi$ and indices $i, j=1, \ldots, d^{\chi}$. In particular, one has $V_{i i}^{\chi}=P_{i}^{\chi}$. A straightforward calculation shows that

$$
\left(V_{i j}^{\chi}\right)^{\dagger}=V_{j i}^{\chi}, \quad V_{i j}^{\chi} V_{k l}^{\chi^{\prime}}=\delta^{\chi \chi^{\prime}} \delta_{j k} V_{i l}^{\chi}
$$

As an immediate consequence, one obtains $V_{i j}^{X} P_{k}^{X}=0$ if $k \neq j$, so that the domain of $V_{i j}^{X}$ reduces naturally to $\operatorname{Im} P_{j}^{X}$ on account of (2.10). Since $\left(V_{i j}^{\chi}\right)^{\dagger} V_{i j}^{X}=V_{j i}^{\chi} V_{i j}^{X}=V_{j j}^{X}=P_{j}^{X}$, it holds that $\operatorname{Ker} V_{i j}^{X}=\operatorname{Ker} P_{j}^{X}$. Similarly, from $V_{i j}^{\chi}\left(V_{i j}^{X}\right)^{\dagger}=V_{i j}^{X} V_{j i}^{X}=V_{i i}^{X}=P_{i}^{X}$, it follows that $\operatorname{Ker}\left(V_{i j}^{\chi}\right)^{\dagger}$ $=\operatorname{Ker} P_{i}^{X}$, and thereby that $\operatorname{Im} V_{i j}^{X}=\left(\operatorname{Ker}\left(V_{i j}^{X}\right)^{\dagger}\right)^{\perp}=\left(\operatorname{Ker} P_{i}^{X}\right)^{\perp}=\operatorname{Im}\left(P_{i}^{X}\right)^{\dagger}=\operatorname{Im} P_{i}^{X}$. Therefore, $V_{i j}^{X}$ becomes a unitary transformation

$$
V_{i j}^{\chi}: \operatorname{Im} P_{j}^{\chi} \rightarrow \operatorname{Im} P_{i}^{\chi}
$$

As a collection of $V_{i j}^{\chi}$, we define another operator $V^{\chi}: \mathcal{H}^{\chi} \otimes \mathcal{H} \rightarrow \mathcal{H}^{\chi} \otimes \mathcal{H}$ by 


$$
V^{\chi}:=d^{\chi} \int_{G} \rho^{\chi}(g) \otimes U(g) d \mu_{G}(g) .
$$

Then Eq. (2.12) implies that

$$
\left(V^{\chi}\right)^{\dagger}=V^{\chi}, \quad V^{\chi} V^{\chi}=d^{\chi} V^{\chi},
$$

which shows that $V^{\chi} / d^{\chi}$ is a projection operator on $\mathcal{H}^{\chi} \otimes \mathcal{H}$. Further, it is easy to verify that $V^{\chi}$ satisfies, for any $h \in G$,

$$
\begin{aligned}
& V^{\chi}\left(\rho^{\chi}(h) \otimes U(h)\right)=V^{\chi}, \\
& \left(\rho^{\chi}(h) \otimes U(h)\right) V^{\chi}=V^{\chi},
\end{aligned}
$$

which implies that

$$
\operatorname{Im} V^{\chi}=\left(\mathcal{H}^{\chi} \otimes \mathcal{H}\right)^{G}:=\left\{\psi \in \mathcal{H}^{\chi} \otimes \mathcal{H} \mid\left(\rho^{\chi}(h) \otimes U(h)\right) \psi=\psi, \forall h \in G\right\}
$$

We call $\left(\mathcal{H}^{\chi} \otimes \mathcal{H}\right)^{G}$ the subspace of invariant vectors of $\mathcal{H}^{\chi} \otimes \mathcal{H}$.

Let $\left\{e_{1}^{\chi}, \ldots, e_{d \chi}^{\chi}\right\}$ be an orthonormal basis of $\mathcal{H}^{\chi}$, which defines an injection $e_{i}^{\chi}: C \rightarrow \mathcal{H}^{\chi}$ by $\lambda \mapsto \lambda e_{i}^{\chi}$ for each $i=1, \ldots, d^{\chi}$. Its adjoint operator $\left(e_{i}^{\chi}\right)^{\dagger}: \mathcal{H}^{\chi} \rightarrow \boldsymbol{C}$ is defined by a surjection $v \mapsto\left\langle e_{i}^{\chi}, v\right\rangle, v \in \mathcal{H}^{\chi}$. The domain of respective maps extends to the tensor product space with $\mathcal{H}$ to give rise to $e_{i}^{\chi}: \mathcal{H} \rightarrow \mathcal{H}^{\chi} \otimes \mathcal{H}$ and $\left(e_{i}^{\chi}\right)^{\dagger}: \mathcal{H}^{\chi} \otimes \mathcal{H} \rightarrow \mathcal{H}$. With these notations, $V_{i j}^{\chi}$ is put in the form $V_{i j}^{\chi}=\left(e_{i}^{\chi}\right)^{\dagger} V^{\chi} e_{j}^{\chi}$. We then introduce an operator by

$$
S_{j}^{\chi}:=\frac{1}{\sqrt{d^{\chi}}} V^{\chi} e_{j}^{\chi}: \mathcal{H} \rightarrow \mathcal{H}^{\chi} \otimes \mathcal{H} .
$$

The adjoint operator is expressed as

$$
\left(S_{j}^{\chi}\right)^{\dagger}:=\frac{1}{\sqrt{d^{\chi}}}\left(e_{j}^{\chi}\right)^{\dagger} V^{\chi}: \mathcal{H}^{\chi} \otimes \mathcal{H} \rightarrow \mathcal{H} .
$$

Then the second relation of (2.15) yields

$$
\left(S_{j}^{\chi}\right)^{\dagger} S_{j}^{\chi}=\left(e_{j}^{\chi}\right)^{\dagger} V^{\chi} e_{j}^{\chi}=P_{j}^{\chi} .
$$

On the other hand, from (2.12), we observe that $V^{\chi} e_{j}^{\chi}\left(e_{j}^{\chi}\right)^{\dagger} V^{\chi}=V^{\chi}$, and thereby obtain

$$
S_{j}^{\chi}\left(S_{j}^{\chi}\right)^{\dagger}=\frac{1}{d^{\chi}} V^{\chi} e_{j}^{\chi}\left(e_{j}^{\chi}\right)^{\dagger} V^{\chi}=\frac{1}{d^{\chi}} V^{\chi} .
$$

Since $P_{j}^{\chi}$ and $V^{\chi} / d^{\chi}$ are projection operators on $\mathcal{H}$ and on $\mathcal{H}^{\chi} \otimes \mathcal{H}$, respectively, Eqs. (2.21) and (2.22) are put together to imply that the restricted map

$$
S_{j}^{\chi}: \operatorname{Im} P_{j}^{\chi} \stackrel{\sim}{\rightarrow} \operatorname{Im} V^{\chi}=\left(\mathcal{H}^{\chi} \otimes \mathcal{H}\right)^{G}
$$

is a unitary transformation. Thus the subspace $\operatorname{Im} P_{j}^{\chi}$ is characterized as $\left(\mathcal{H}^{\chi} \otimes \mathcal{H}\right)^{G}$.

We turn to the Hamiltonian $H$ acting on $\mathcal{H}$. It appears that $H$ is extended to an operator $\operatorname{id}_{\mathcal{H} \chi} \otimes H$ on $\mathcal{H}^{\chi} \otimes \mathcal{H}$. Since $H$ commutes with $U(g)$ for each $g \in G$, the extended operator $\operatorname{id}_{\mathcal{H} \chi}$ $\otimes H$ commutes with $V^{\chi}$ and with $S_{j}^{\chi}$ as well, so that one has

$$
S_{j}^{\chi} H=\left(\operatorname{id}_{\mathcal{H} \chi} \otimes H\right) S_{j}^{\chi} .
$$


It then turns out that the reduced system $\left(\operatorname{Im} P_{j}^{\chi}, H\right)$ is identified with $\left(\left(\mathcal{H}^{\chi} \otimes \mathcal{H}\right)^{G}, \operatorname{id}_{\mathcal{H}} \otimes \otimes H\right)$, which will be investigated in the following sections in detail.

Before investigation into the reduced system, we wish to consider how the action of $G$ is decomposed according to the decomposition of $\mathcal{H}$. In the below, the dual space $\left(\mathcal{H}^{X}\right)^{*}$ to $\mathcal{H}^{X}$ is taken as $\left(\mathcal{H}^{x}\right)^{*}:=\operatorname{Hom}\left(\mathcal{H}^{x} ; \boldsymbol{C}\right)$, irrespective of the inner product in $\mathcal{H}^{x}$. Then the map $\sqrt{d^{\chi}}\left(S_{j}^{\chi}\right)^{\dagger}=\left(e_{j}^{\chi}\right)^{\dagger} V^{\chi}: \mathcal{H}^{\chi} \otimes \mathcal{H} \rightarrow \mathcal{H}$ can be viewed as a map $T_{j}^{\chi}: \mathcal{H} \rightarrow\left(\mathcal{H}^{\chi}\right)^{*} \otimes \mathcal{H}$ in the manner as follows: Using the identification End $\mathcal{H}^{X} \cong\left(\mathcal{H}^{X}\right)^{*} \otimes \mathcal{H}^{X}$, we may regard the map $V^{X}: \mathcal{H}^{X} \otimes \mathcal{H}$ $\rightarrow \mathcal{H}^{\chi} \otimes \mathcal{H}$ as a map $V^{\chi}: \mathcal{H} \rightarrow\left(\mathcal{H}^{\chi}\right)^{*} \otimes \mathcal{H}^{\chi} \otimes \mathcal{H}$. Combining $\operatorname{id}_{(\mathcal{H} X) *} \otimes\left(e_{j}^{\chi}\right)^{\dagger}:\left(\mathcal{H}^{\chi}\right)^{*} \otimes \mathcal{H}^{\chi} \otimes \mathcal{H}$ $\rightarrow\left(\mathcal{H}^{\chi}\right)^{*} \otimes \mathcal{H}$ with $V^{\chi}$, we can express $T_{j}^{\chi}$ as the map

$$
T_{j}^{\chi}=\left(\operatorname{id}_{(\mathcal{H} \chi)^{*}} \otimes\left(e_{j}^{\chi}\right)^{\dagger}\right) V^{\chi}: \mathcal{H} \rightarrow\left(\mathcal{H}^{\chi}\right)^{*} \otimes \mathcal{H} .
$$

Then it can be verified that

$$
\left(T_{j}^{\chi}\right)^{\dagger} T_{j}^{\chi}=\sum_{k} P_{k}^{\chi}, \quad T_{j}^{\chi}\left(T_{j}^{\chi}\right)^{\dagger}=\operatorname{id}_{(\mathcal{H} \chi) *} \otimes P_{j}^{\chi},
$$

where $\left(T_{j}^{\chi}\right)^{\dagger}:\left(\mathcal{H}^{\chi}\right)^{*} \otimes \mathcal{H} \rightarrow \mathcal{H}$ is the adjoint operator. From this, it follows that $T_{j}^{\chi}$ yields a unitary transformation

$$
T_{j}^{\chi}: \bigoplus_{k} \operatorname{Im} P_{k}^{\chi} \stackrel{\sim}{\longrightarrow}\left(\mathcal{H}^{\chi}\right)^{*} \otimes \operatorname{Im} P_{j}^{\chi} .
$$

Then the right invariance of $V^{\chi}$ under the $G$ action, expressed in (2.16), makes the following diagram commutative,

$$
\begin{array}{rlc}
\mathcal{H} & =\bigoplus_{\chi, i} \operatorname{Im} P_{i}^{\chi} \stackrel{T_{j}^{\chi}}{\longrightarrow} & \left(\mathcal{H}^{\chi}\right)^{*} \otimes \operatorname{Im} P_{j}^{\chi} \\
U(h) \downarrow & & \downarrow^{t} \rho^{\chi}\left(h^{-1}\right) \otimes \mathrm{id} \\
\mathcal{H} & =\bigoplus_{\chi, i} \operatorname{Im} P_{i}^{\chi} \stackrel{T_{j}^{\chi}}{\longrightarrow} & \left(\mathcal{H}^{\chi}\right)^{*} \otimes \operatorname{Im} P_{j}^{\chi},
\end{array}
$$

where ${ }^{t} \rho^{\chi}\left(h^{-1}\right)=\overline{\rho^{\chi}(h)}$ is the contragredient representation of $G$ on $\left(\mathcal{H}^{\chi}\right)^{*}$. As for the action of the Hamiltonian $H$, we obtain the following commutative diagram accordingly:



The commutative diagrams (2.28) and (2.29) show that the decomposition of the representation $(G, \mathcal{H}, U)$ is compatible with the spectral resolution of $H$.

\section{Equivariant functions}

The general reduction method introduced in the previous section applies to a quantum system on a configuration space $M$ which admits the action of a compact Lie group $G$.

Suppose that a compact Lie group $G$ acts on a differentiable manifold $M$ by diffeomorphisms, namely, we are given a $C^{\infty}$ map $G \times M \rightarrow M$ satisfying $e x=x$ and $(g h) x=g(h x)$ for any $x \in M$ and any $g, h \in G$ with the identity element $e \in G$. Then $M$ is called a $G$-manifold. In addition, 
suppose that $M$ is equipped with a measure $\mu_{M}$ which is invariant under the action of $G$. Let $\mathcal{H}$ $=L_{2}(M)$ be the space of square integrable functions on $M$ with respect to the measure $\mu_{M}$. The group $G$ is represented on $\mathcal{H}=L_{2}(M)$ by unitary operators $U(h), h \in G$, through

$$
(U(h) f)(x):=f\left(h^{-1} x\right), f \in L_{2}(M) .
$$

Then the reduction method applies to $\mathcal{H}=L_{2}(M)$ to yield the Hilbert subspace $\operatorname{Im} P_{j}^{\chi}$. The unitary transformation $S_{j}^{\chi}$ given in (2.23) allows us to identify $\operatorname{Im} P_{j}^{\chi}\left(\subset L_{2}(M)\right)$ with $\left(\mathcal{H}^{X}\right.$ $\left.\otimes L_{2}(M)\right)^{G}\left(\subset \mathcal{H}^{X} \otimes L_{2}(M)\right)$. The space $\mathcal{H}^{X} \otimes L_{2}(M)$ can be identified with the Hilbert space, $L_{2}\left(M ; \mathcal{H}^{X}\right)$, of square integrable $\mathcal{H}^{X}$-valued functions on $M$;

$$
L_{2}\left(M ; \mathcal{H}^{\chi}\right):=\left\{\psi: M \rightarrow \mathcal{H}^{\chi} \mid \int_{M}\|\psi(x)\|^{2} d \mu_{M}(x)<\infty\right\}
$$

which is equipped with the inner product,

$$
\langle\phi, \psi\rangle:=\int_{M}\langle\phi(x), \psi(x)\rangle d \mu_{M}(x), \quad \phi, \psi \in L_{2}\left(M ; \mathcal{H}^{\chi}\right),
$$

where $\langle\phi(x), \psi(x)\rangle$ denotes the inner product in $\mathcal{H}^{x}$. Then, the condition given in (2.18) along with (2.30) implies that $\psi \in\left(\mathcal{H}^{X} \otimes L_{2}(M)\right)^{G}$, when viewed as an $\mathcal{H}^{X}$-valued function, satisfies

$$
\psi(h x)=\rho^{\chi}(h) \psi(x), \quad h \in G,
$$

which shows that $\psi$ is equivariant under the $G$-action. We conclude therefore that the reduced Hilbert space $\operatorname{Im} P_{j}^{X}$ is identified with the space of square integrable equivariant functions, which we denote by $L_{2}\left(M ; \mathcal{H}^{\chi}\right)^{G}$,

$$
\left(\mathcal{H}^{\chi} \otimes L_{2}(M)\right)^{G} \cong L_{2}\left(M ; \mathcal{H}^{\chi}\right)^{G}=\left\{\psi \in L_{2}\left(M ; \mathcal{H}^{\chi}\right) \mid \psi(g x)=\rho^{\chi}(g) \psi(x), g \in G\right\} .
$$

Here we have to note that according to the decomposition (2.10) along with (2.23), (2.27), and (2.34), the Hilbert space $L_{2}(M)$ is decomposed into

$$
L_{2}(M) \cong \oplus_{\chi}\left(\left(\mathcal{H}^{\chi}\right)^{*} \otimes L_{2}\left(M ; \mathcal{H}^{\chi}\right)^{G}\right) .
$$

So far we have characterized the reduced Hilbert space on the $G$-manifold $M$. We now have to specify the Hamiltonian on $L_{2}(M)$ and to reduce it. We will take a Hamiltonian as defined to be the sum of the Laplacian on $M$ and a potential energy function on $M$. In the succeeding sections, we will study the geometric structure of $M$ in order to analyze the Laplacian on $M$ according to the decomposition (2.35).

\section{GEOMETRIC SETTING ON G-MANIFOLDS}

\section{A. Stratification of G-manifolds}

According to Davis, ${ }^{23} G$-manifolds can be viewed as collections of fiber bundles. We here make a brief review of his idea in a suitable form for our application. For more rigorous definitions, see the literature. ${ }^{24}$

Let $M$ be a $G$-manifold. For a point $x \in M$, we denote the isotropy subgroup at $x$ and the G-orbit of $x$ by $G_{x}:=\{g \in G \mid g x=x\}$ and by $\mathcal{O}_{x}:=\{g x \mid g \in G\}$, respectively. Then one has $\mathcal{O}_{x}$ $\cong G / G_{x}$.

Take $G_{x}$ and $G_{y}$ for two points $x, y \in M$. If $G_{x}$ is conjugate to $G_{y}$ by an inner automorphism $A_{g}: G \rightarrow G ; h \mapsto g h g^{-1}$ with some $g \in G ; G_{y}=g \cdot G_{x} \cdot g^{-1}$, then the orbits $\mathcal{O}_{x}$ and $\mathcal{O}_{y}$ are diffeomorphic to each other by the correspondence induced by $A_{g}$. Of course, if $y$ is in the orbit $\mathcal{O}_{x}$, namely, if there exists some $g \in G$ such that $y=g x$, then $G_{y}$ is conjugate to $G_{x}$ by $A_{g}$. The conjugacy class of $G_{x}$ is called the orbit type of $\mathcal{O}_{x}$ and denoted by $\left[G_{x}\right]$ or $\tau$. We say that the 
point $x$ itself also has the orbit type $\tau$, if $\mathcal{O}_{x}$ has the orbit type $\tau$. Let $\mathcal{T}(M)$ denote the set of all the orbit types appearing in $M ; \mathcal{T}(M):=\left\{\left[G_{x}\right] \mid x \in M\right\}$. For each $\tau \in \mathcal{T}(M)$, we denote a representative of the conjugacy class by $G_{\tau} \in \tau$.

One can introduce a partial order on $\mathcal{T}(M)$ as follows: We say that $\tau_{1}$ is lower than $\tau_{2}$ $\left(\tau_{1}, \tau_{2} \in \mathcal{T}(M)\right), \tau_{1} \leqslant \tau_{2}$, if there are representatives $G_{\tau_{1}} \in \tau_{1}$ and $G_{\tau_{2}} \in \tau_{2}$ such that $G_{\tau_{1}} \supset G_{\tau_{2}}$. An orbit $\mathcal{O}_{x}$ is called maximal if its orbit type $\tau$ is maximal with respect to this order. Orbits which are not maximal are called singular. We say also that a point $x$ is maximal or singular according to whether the orbit type of $\mathcal{O}_{x}$ is maximal or singular. For each orbit type $\tau \in \mathcal{T}(M)$, we denote by $M_{\tau}:=\left\{x \in M \mid G_{x} \in \tau\right\}$ the set of points with the same orbit type $\tau$, which becomes a smooth manifold. Thus $M$ is stratified into a collection of the smooth submanifolds $M_{\tau}, M=\amalg_{\tau} M_{\tau}$, which is partially ordered by the system of orbit types $(\mathcal{T}(M), \leqslant)$. Each manifold $M_{\tau}$ is called a stratum.

The set of orbits $Q:=M / G$ becomes a topological space with respect to the quotient topology which is defined by demanding that the canonical projection map $\pi: M \rightarrow M / G$ is continuous. The space $Q$ is called an orbit space. A point $q \in Q$ is also classified by the orbit type of $\pi^{-1}(q)$. The point $q$ is said to be maximal or singular according to whether the orbit is maximal or singular. The $Q$ inherits differentiable structure from $M$, if and only if all the orbits have the same orbit type. If otherwise, $Q$ is stratified into a collection of smooth manifolds of various dimensions. Setting $Q_{\tau}:=\pi\left(M_{\tau}\right)$, one has $Q=\amalg_{\tau} Q_{\tau}$. The restriction of $\pi: M \rightarrow Q$ onto each stratum $\pi_{\tau}: M_{\tau}$ $\rightarrow Q_{\tau}$ defines a fiber bundle with fiber $G / G_{\tau}$. We then call the pentaplet $(M, G, Q, \pi, \mathcal{T}(M))$ a stratified bundle.

If every point of $M$ has the same orbit type, $\pi: M \rightarrow Q$ is nothing but a usual fiber bundle. In particular, if the group $G$ acts on $M$ freely, namely, $G_{x}=\{e\}$ for all $x \in M$, then $\pi: M \rightarrow Q$ becomes a principal fiber bundle with structure group $G$. In this sense, we may regard the $G$-manifold as a generalization of fiber bundles although the base space $Q=M / G$ is not a smooth manifold. Mechanics of molecules provides an example of a stratified bundle that is not a fiber bundle, as shown in the later section.

According to the principal orbit theorem, ${ }^{25,23}$ if the orbit space $Q=M / G$ is connected, there is the maximum orbit type in $\mathcal{T}(M)$ with respect to the order $\leqslant$. Although the maximum orbit type is also named the principal orbit type in Refs. 25 and 23, we will call it maximum in this paper. We assume that $Q$ is connected and that the maximum orbit exists. We denote the maximum orbit type by $\mu$. Moreover, the principal orbit theorem ${ }^{25,23}$ states that the maximum stratum $M_{\mu}$ is open and dense in $M$. Thus the set $M_{\text {sing }}:=M-M_{\mu}$ of all the singular points coincides with the boundary $\partial M_{\mu}$. The image of $M_{\mu}$ and $\partial M_{\mu}$ by the projection $\pi$ are denoted by $Q_{\mu}:=\pi\left(M_{\mu}\right)$ and $\partial Q_{\mu}:=\pi\left(\partial M_{\mu}\right)$, respectively. We put $\operatorname{dim} M=m$ and $\operatorname{dim} Q_{\mu}=n=m-p$. Then the dimension of the maximum orbit is $\operatorname{dim} G / G_{\mu}=p$.

Though the orbit space $Q$ is not a manifold, one can speak of differentiability of functions on $Q$. A function of $\varphi: Q \rightarrow \boldsymbol{R}$ is called of $C^{r}$ class when $\varphi^{\circ} \pi: M \rightarrow \boldsymbol{R}$ is a differentiable function of $C^{r}$ class. Clearly, $\varphi^{\circ} \pi$ is a $G$-invariant function on $M$. Conversely, any $G$-invariant function $f$ on $M$ is identified with a function on $Q$. We denote the space of $G$-invariant functions on $M$ of $C^{r}$ class by $C^{r}(M)^{G}=\{f: M \rightarrow \boldsymbol{R} \mid f(g x)=f(x), \forall g \in G, \forall x \in M\}$.

A tangent vector $X$ to $M$ at $x$ is usually defined as a differential operator acting on $C^{\infty}(M)$; $X: C^{\infty}(M) \rightarrow \boldsymbol{R}$. A tangent vector on $Q$ is defined as follows: We define an equivalence relation $\sim$ in the tangent vector space $T_{x} M$ by stating that $X \sim Y$ if $X f=Y f$ for all $f \in C^{\infty}(M)^{G}$. The equivalence class of $X$ is denoted by $\pi_{*}(X)$, which defines a linear map $\pi_{*}(X): C^{\infty}(M)^{G} \rightarrow \boldsymbol{R}$. The set $T_{q} Q:=\left\{\pi_{*}(X) \mid X \in T_{x} M, \pi(x)=q\right\}$ becomes a vector space through the structure of the vector space $T_{x} M$ and is called a tangent vector space at $q \in Q$.

\section{B. Stratified connection}

Let $(M, G, Q, \pi, \mathcal{T}(M))$ be the stratified bundle defined above. If $G$ acts on $M$ freely, the stratified bundle becomes a principal fiber bundle. Although the concept of connection is usually defined on principal fiber bundles, we would here like to define extended connections on stratified bundles. 
Let us call a subspace $V_{x}:=T_{x} \mathcal{O}_{x}$ of $T_{x} M$ a vertical subspace at $x \in M$. The action of $g$ $\in G: M \rightarrow M ; x \mapsto g x$ induces an action $g_{*}: T_{x} M \rightarrow T_{g x} M$ by differentiation. The complement $H_{x}$ of $V_{x}$ is called a horizontal subspace. If the direct sum decomposition $T_{x} M=V_{x} \oplus H_{x}$ is smooth and if the family $\left\{H_{x}\right\}_{x \in M}$ satisfies the invariance; $H_{g x}=g_{*} H_{x}$, the decomposition $T_{x} M=V_{x}$ $\oplus H_{x}$ defines a connection. However, we should note that $\operatorname{dim} V_{x}=\operatorname{dim} \mathcal{O}_{x}$ and $\operatorname{dim} H_{x}=\operatorname{dim} M$ $-\operatorname{dim} \mathcal{O}_{x}$ jump suddenly when the point $x$ passes singular points. Thus the smoothness of the decomposition $T_{x} M=V_{x} \oplus H_{x}$ must be required on each stratum $M_{\tau}$, so that one understands that for any smooth vector field $X$ which is decomposed into $X(x)=X^{V}(x)+X^{H}(x)$ according to $T_{x} M=V_{x} \oplus H_{x}$, the components $X^{V}$ and $X^{H}$ are also smooth on each $M_{\tau}$. Then the assignment $x \mapsto H_{x}$ for each $x \in M$ is called a stratified connection over the stratified bundle $\pi: M \rightarrow Q$.

The decomposition $T_{x} M=V_{x} \oplus H_{x}$ induces a decomposition of the dual space $T_{x}^{*} M=V_{x}^{*}$ $\oplus H_{x}^{*}$ with $V_{x}^{*}:=\left\{\phi \in T_{x}^{*} M \mid \phi(v)=0, \forall v \in H_{x}\right\}$ and $H_{x}^{*}=\left\{\psi \in T_{x}^{*} M \mid \psi(u)=0, \forall u \in V_{x}\right\}$.

Let $\mathbf{g}$ and $\mathbf{g}_{x}$ denote the Lie algebras of $G$ and $G_{x}$, respectively. The relation $\mathbf{g}_{g x}=\operatorname{Ad}_{g} \mathbf{g}_{x}$ is an immediate consequence of $G_{g x}=A_{g} G_{x}$. The group action $G \times M \rightarrow M ;(g, x) \mapsto g x$ gives rise to vector fields $\mathbf{g} \times M \rightarrow T M ;(\xi, x) \mapsto \xi_{M}(x)$ as infinitesimal transformations. Fixing a point $x \in M$, one obtains a linear map $\theta_{x}: \mathbf{g} \rightarrow T_{x} M ; \theta_{x}(\xi)=\xi_{M}(x)$. It then follows that $\operatorname{Ker} \theta_{x}=\mathbf{g}_{x}$ and $\operatorname{Im} \theta_{x}$ $=V_{x}$, and hence that $\theta_{x}: \mathbf{g} \rightarrow T_{x} M$ induces an isomorphism $\widetilde{\theta}_{x}: \mathbf{g} / \mathbf{g}_{x} \underset{\rightarrow}{\rightarrow} V_{x}$.

The connection defined above is described in term of differential forms. A connection form $\omega$ is defined as the composition of the projection $T_{x} M=V_{x} \oplus H_{x} \rightarrow V_{x}$ and the inverse map $\left(\widetilde{\theta}_{x}\right)^{-1}: V_{x} \stackrel{\sim}{\rightarrow} \mathbf{g} / \mathbf{g}_{x}$,

$$
\omega_{x}: T_{x} M=V_{x} \oplus H_{x} \rightarrow V_{x} \stackrel{\sim}{\rightarrow} \mathbf{g} / \mathbf{g}_{x}
$$

at each point $x \in M$. The form $\omega$ is thus a one-form which takes values in quotient spaces of the Lie algebra $\mathbf{g}$. It has the following properties:

$$
\begin{gathered}
\omega\left(\theta_{x}(\xi)\right) \equiv \xi\left(\bmod \mathbf{g}_{x}\right), \quad \xi \in \mathbf{g}, \\
\left(g^{*} \omega\right)_{x}=\operatorname{Ad}_{g} \omega_{x}, \quad g \in G,
\end{gathered}
$$

where $g^{*}$ is the pull-back associated with the map $g_{*}: T_{x} M \rightarrow T_{g x} M$ and $\operatorname{Ad}_{g}$ is to be understood as a map $\operatorname{Ad}_{g}: \mathbf{g} / \mathbf{g}_{x} \rightarrow \mathbf{g} / \mathbf{g}_{g x}$. To verify (3.3), we need the formula that $\theta_{g x}\left(\operatorname{Ad}_{g} \xi\right)=g_{*}\left(\theta_{x}(\xi)\right)$ for $\xi \in \mathbf{g}$ and that $\left(\widetilde{\theta}_{g x}\right)^{-1} \circ g_{*}=\operatorname{Ad}_{g}\left(\widetilde{\theta}_{x}\right)^{-1}$. The properties (3.2) and (3.3) are generalization of the well-known defining properties of usual connection forms. It is also noted that the composition map

$$
\widetilde{\theta_{x}^{\circ}} \omega_{x}: T_{x} M=V_{x} \oplus H_{x} \rightarrow V_{x}
$$

is a projection, and that Eq. (3.2) is equivalently written as $\omega_{x} \circ \widetilde{\theta}_{x}=\mathrm{id}_{\mathbf{g} / \mathbf{g}_{x}}$.

\section{Equivariant forms}

Let $\psi \in C^{\infty}\left(M, \mathcal{H}^{\chi}\right)^{G}$ be a smooth equivariant function, that is, one has $\psi(g x)=\rho^{\chi}(g) \psi(x)$ for $g \in G$ (see (2.33)). If a point $x \in M$ carries a nontrivial isotropy group $G_{x}$, then the value of $\psi(x)$ becomes invariant under the action of $G_{x} ; \psi(x)=\rho^{\chi}(g) \psi(x)$ for $g \in G_{x}$. For a subgroup $G_{1} \subset G$, we here define $\left(\mathcal{H}^{X}\right)^{G_{1}}$ to be a maximum subspace of invariant vectors under the action of $G_{1}$, that is, $\left(\mathcal{H}^{\chi}\right)^{G_{1}}:=\left\{v \in \mathcal{H}^{\chi} \mid \rho^{\chi}(g) v=v, \forall g \in G_{1}\right\}$. With this notation, we then have $\psi(x) \in\left(\mathcal{H}^{\chi}\right)^{G_{x}}$.

Properties of subspaces of invariant vectors are worth remarking. One has obviously $\left(\mathcal{H}^{\chi}\right)^{\{e\}}=\mathcal{H}^{\chi}$. Further, one obtains $\left(\mathcal{H}^{\chi}\right)^{G}=\{0\}$, if and only if there is no nontrivial invariant vector. To a sequence of subgroups $\{e\} \subset G_{1} \subset G_{2}(\subset G)$, there corresponds a sequence of sub- 
spaces $\mathcal{H}^{\chi} \supset\left(\mathcal{H}^{\chi}\right)^{G_{1}} \supset\left(\mathcal{H}^{\chi}\right)^{G_{2}}(\supset\{0\})$. For conjugate subgroups $G_{1}$ and $A_{g} G_{1}$, it holds that $\left(\mathcal{H}^{\chi}\right)^{A_{g} G_{1}}=\rho^{\chi}(g)\left(\mathcal{H}^{\chi}\right)^{G_{1}}$. In particular, on an orbit $\mathcal{O}_{x}$, one has $\left(\mathcal{H}^{\chi}\right)^{G_{g x}}=\rho^{\chi}(g)\left(\mathcal{H}^{\chi}\right)^{G_{x}}$ on account of $G_{g x}=A_{g} G_{x}$.

Like equivariant functions, we can define equivariant differential forms in a similar manner. An equivariant $k$-form is defined as an $\mathcal{H}^{\chi}$-valued differential $k$-form $\alpha: \wedge^{k} T M \rightarrow \mathcal{H}^{\chi}$ satisfying $g^{*} \alpha=\rho^{\chi}(g) \alpha$ for any $g \in G$. When an equivariant $k$-form $\alpha$ further satisfies $i\left(\xi_{M}\right) \alpha=0$ for any $\xi \in \mathbf{g}$, it is called an equivariant horizontal $k$-form. While the set of all the differential $k$-form on $M$ is denoted by $\Omega^{k}(M)$, the set of all the $\mathcal{H}^{\chi}$-valued differential $k$-forms and the set of all the equivariant horizontal $k$-forms are denoted by $\mathcal{H}^{\chi} \otimes \Omega^{k}(M) \cong \Omega^{k}\left(M ; \mathcal{H}^{\chi}\right)$ and $\Omega_{H}^{k}\left(M ; \mathcal{H}^{\chi}\right)^{G}$, respectively.

At each point $x \in M$ carrying a nontrivial isotropy group $G_{x}$, the equivariant horizontal $k$-form $\alpha$ takes a restricted range as well as equivalent functions. It turns out that $\alpha_{x}\left(X_{1}, \ldots, X_{k}\right) \in\left(\mathcal{H}^{\chi}\right)^{G_{x}}$ for any $X_{1}, \ldots, X_{k} \in T_{x} M$ when $G_{x}$ is connected. The proof is given below: Let $L_{X}$ denote the Lie derivation by a vector field $X$ on $M$, and $\rho_{*}^{\chi}$ denote a representation of the Lie algebra $\mathbf{g}$ on $\mathcal{H}^{\chi}$ induced by differentiation of the representation $\rho^{\chi}$ of $G$. The defining property of the equivariant form $g^{*} \alpha=\rho^{\chi}(g) \alpha$ for $g \in G$ is differentiated to give $L_{\xi_{M}} \alpha$ $=\rho_{*}^{\chi}(\xi) \alpha$ for $\xi \in \mathbf{g}$. This equation and the horizontal condition $i\left(\xi_{M}\right) \alpha=0$ for $\xi \in \mathbf{g}$ are put together with the Cartan formula $L_{\xi_{M}} \alpha=i\left(\xi_{M}\right) d \alpha+d i\left(\xi_{M}\right) \alpha$ to provide

$$
\rho_{*}^{\chi}(\xi) \alpha=i\left(\xi_{M}\right) d \alpha, \quad \xi \in \mathbf{g} .
$$

If $\xi \in \mathbf{g}_{x}$, one has $\xi_{M}(x)=0$ and hence $\rho_{*}^{\chi}(\xi) \alpha_{x}=0$ from (3.5), which implies that $\rho^{\chi}(g) \alpha_{x}$ $=\alpha_{x}$ for $g \in G_{x}$ if $G_{x}$ is connected. Thus we conclude that $\alpha_{x} \in\left(\mathcal{H}^{\chi}\right)^{G_{x}}$.

\section{Associated vector bundles}

So far we have discussed equivariant functions and forms on the stratified bundle. We now wish to define vector bundles associated with the stratified bundle, like vector bundles associated with principal fiber bundles.

Let us define an equivalence relation $\sim$ in $M \times \mathcal{H}^{\chi}$ by the relation $(x, v) \sim\left(g x, \rho^{\chi}(g) v\right)$ with $g \in G$. Let $[x, v]$ denote the equivalence class with a representative $(x, v)$. Then the vector bundle associated with the stratified bundle $(M, G, Q, \pi, \mathcal{T}(M))$ by a representation $\left(\mathcal{H}^{\chi}, \rho^{\chi}\right)$ is defined to be the quotient space,

$$
M \times{ }_{\rho} \chi \mathcal{H}^{\chi}:=\left(\amalg_{x \in M}\left(\{x\} \times\left(\mathcal{H}^{\chi}\right)^{G_{x}}\right)\right) / \sim
$$

The projection map $M \times \mathcal{H}^{\chi} \rightarrow M$ naturally induces a projection map $\pi_{\rho} \chi: M \times{ }_{\rho} \mathcal{H}^{\chi} \rightarrow Q$; $[x, v] \mapsto \pi(x)$. Further, each point $x \in M$ defines an isomorphism $\tilde{x}:\left(\mathcal{H}^{\chi}\right)^{G_{x}} \rightarrow \pi_{\rho \chi}^{-1}(\pi(x))=\mathcal{O}_{x}$ $\times_{\rho} \chi \mathcal{H}^{\chi}$ by $v \mapsto \tilde{x}(v):=[x, v]$. Note that, for each stratum $Q_{\tau}, \pi_{\rho^{\chi}}^{-1}\left(Q_{\tau}\right)$ is a vector bundle over $Q_{\tau}$ with fiber $\left(\mathcal{H}^{\chi}\right)^{G}$, so that one has $M \times{ }_{\rho} \chi \mathcal{H} \mathcal{H}^{\chi}=\amalg_{\tau} \pi_{\rho \chi}^{-1}\left(Q_{\tau}\right)$. In this sense, we may call $M$ $\times{ }_{\rho} \chi \mathcal{H}^{\chi}$ a stratified vector bundle. However, we will refer to it as the associated vector bundle for simplicity. Moreover, each fiber $\pi_{\rho^{\chi}}^{-1}(q)$ at $q \in Q$ inherits an inner product from $\mathcal{H}^{\chi}$; for $\eta, \eta^{\prime}$ $\in \pi_{\rho^{\chi}}^{-1}(q)$, the inner product $\left\langle\eta, \eta^{\prime}\right\rangle$ is defined to be

$$
\left\langle\eta, \eta^{\prime}\right\rangle:=\left\langle\tilde{x}^{-1}(\eta), \tilde{x}^{-1}\left(\eta^{\prime}\right)\right\rangle,
$$

where the RHS is the inner product defined on $\mathcal{H}^{x}$. It is easy to verify that the RHS is independent of the choice of $x \in \pi^{-1}(q)$.

A section of the associated vector bundle is a map $\sigma: Q \rightarrow M \times{ }_{\rho} \chi \mathcal{H}^{\chi}$ satisfying $\pi_{\rho} \chi^{\circ} \sigma$ $=\mathrm{id}_{Q}$. An equivariant function $\psi$ defines a section $\psi^{b}$ of the associated vector bundle through $\psi^{b}(\pi(x))=[x, \psi(x)]=(\tilde{x} \circ \psi)(x)$. The $\psi^{b}(q)$ is well-defined. In fact, $\psi^{b}(\pi(g x))=[g x, \psi(g x)]$ $=\left[g x, \rho^{\chi}(g) \psi(x)\right]=[x, \psi(x)]=\psi^{b}(\pi(x))$ for $g \in G$. Conversely, a section $\sigma$ defines an equivariant function $\sigma^{\sharp}$ through $\sigma^{\sharp}(x)=\left(\tilde{x}^{-1} \circ \sigma^{\circ} \pi\right)(x)$. Thus we can identify sections of the associated 
vector bundle with equivariant functions. When the corresponding equivariant function $\sigma^{\sharp}$ is differentiable as an $\mathcal{H}^{\chi}$-valued function on $M$, the section $\sigma$ is called differentiable, while $Q$ is not a manifold in general. The set of all of the differentiable sections of $M \times{ }_{\rho} \chi \mathcal{H}^{\chi}$ is denoted by $\Gamma\left(M \times{ }_{\rho \chi} \mathcal{H}^{\chi}\right)$. Thus the space of sections $\Gamma\left(M \times{ }_{\rho} \mathcal{H}^{\chi}\right)$ is in one-to-one correspondence with the space of equivariant functions $C^{\infty}\left(M ; \mathcal{H}^{X}\right)^{G}$.

To associate equivariant horizontal $k$-forms with certain sections, we should set up another vector bundle. The action of $g \in G$ on the space of one-forms, $\left(g^{-1}\right)^{*}: T_{x}^{*} M \rightarrow T_{g x}^{*} M$, induces the action on the space of horizontal $k$-forms, $\left(g^{-1}\right)^{*}: \wedge^{k} H_{x}^{*} \rightarrow \wedge^{k} H_{g x}^{*}$. This action is extended to that on the space of $\mathcal{H}^{\chi}$-valued horizontal $k$-forms $\wedge^{k} H^{*} \otimes \mathcal{H}^{\chi}$ by the linear map generated by

$$
\phi \otimes v \in \wedge^{k} H_{x}^{*} \otimes \mathcal{H}^{\chi} \mapsto \widetilde{\rho^{\chi}}(g)(\phi \otimes v):=\left(g^{-1}\right)^{*} \phi \otimes \rho^{\chi}(g) v \in \wedge^{k} H_{g x}^{*} \otimes \mathcal{H}^{\chi} .
$$

We then take a subspace of invariant vectors

$$
\left(\wedge^{k} H_{x}^{*} \otimes \mathcal{H}^{\chi}\right)^{G_{x}}:=\left\{\zeta \in \wedge^{k} H_{x}^{*} \otimes \mathcal{H}^{\chi} \mid \widetilde{\rho^{\chi}}(g) \zeta=\zeta, \forall g \in G_{x}\right\}
$$

and define an associated vector bundle, like (3.6), through

$$
M \times \widetilde{\rho \chi}\left(\wedge^{k} H^{*} \otimes \mathcal{H}^{\chi}\right):=\left(\amalg_{x \in M}\left(\{x\} \times\left(\wedge^{k} H_{x}^{*} \otimes \mathcal{H}^{\chi}\right)^{G_{x}}\right) / \sim,\right.
$$

where the equivalent relation $\sim$ in $M \times\left(\wedge^{k} H^{*} \otimes \mathcal{H}^{\chi}\right)$ is defined as $(x, \zeta) \sim\left(g x, \widetilde{\rho^{\chi}}(g) \zeta\right)$. A projection map $\pi_{\vec{\rho} \chi}: M \times \overrightarrow{\rho x}\left(\wedge^{k} H^{*} \otimes \mathcal{H}^{\chi}\right) \rightarrow Q$ is defined naturally as $[x, \zeta] \mapsto \pi(x)$. The space of smooth sections $\Gamma\left(M \times \overrightarrow{\rho \chi}\left(\wedge^{k} H^{*} \otimes \mathcal{H}^{\chi}\right)\right)$ is also in one-to-one correspondence with the space of equivariant horizontal forms $\Omega_{H}^{k}\left(M ; \mathcal{H}^{X}\right)^{G}$.

\section{E. Covariant derivative}

Covariant derivatives of $k$-forms on the stratified bundle can be defined like those on the principal fiber bundle. Covariant derivation is a linear map $D: \Omega_{H}^{k}\left(M ; \mathcal{H}^{\chi}\right)^{G} \rightarrow \Omega_{H}^{k+1}\left(M ; \mathcal{H}^{\chi}\right)^{G}$ defined through

$$
D \alpha\left(X_{1}, \ldots, X_{k+1}\right)=d \alpha\left(X_{1}^{H}, \ldots, X_{k+1}^{H}\right), \quad X_{i} \in T_{x} M, i=1, \ldots, k+1,
$$

for $\alpha \in \Omega_{H}^{k}\left(M ; \mathcal{H}^{\chi}\right)^{G}$, where $X_{i}^{H}$ is the horizontal component of $X_{i}=X_{i}^{V}+X_{i}^{H}$.

The covariant derivative $D \alpha$ can be expressed by using the connection form $\omega$. To any $X^{V}$ $\in V_{x}$, there corresponds an element $\xi \in \mathbf{g}$ such that $X^{V}=\xi_{M}(x)$ uniquely modulo $\mathbf{g}_{x}$. By the definition of the connection form $\omega$, we then have $\xi \equiv \omega\left(X^{V}\right) \equiv \omega(X)\left(\bmod \mathbf{g}_{x}\right)$. With the help of (3.5), one has $i\left(X^{V}\right) d \alpha=i\left(\xi_{M}\right) d \alpha=\rho_{*}^{X}(\xi) \alpha=\rho_{*}^{X}(\omega(X)) \alpha$. The last equality holds well in spite of the ambiguity in the value of $\omega(X)$ since $\rho_{*}^{X}(\zeta) \alpha_{x}=0$ for $\zeta \in \mathbf{g}_{x}$. Putting together these equations results in

$$
\begin{aligned}
D \alpha\left(X_{1}, \ldots, X_{k+1}\right)= & d \alpha\left(X_{1}-X_{1}^{V}, \ldots, X_{k+1}-X_{k+1}^{V}\right) \\
= & d \alpha\left(X_{1}, \ldots, X_{k+1}\right) \\
& +\sum_{i=1}^{k+1}(-1)^{i} \rho_{*}^{\chi}\left(\omega\left(X_{i}\right)\right) \alpha\left(X_{1}, \ldots, \widehat{X}_{i}, \ldots, X_{k+1}\right),
\end{aligned}
$$

where $\widehat{X}_{i}$ means the removing of $X_{i}$. Thus our result is expressed as

$$
D \alpha=d \alpha-\rho_{*}^{\chi}(\omega) \wedge \alpha .
$$

We should note that smoothness of the covariant derivative is ensured only within each stratum $M_{\tau}$. 


\section{REDUCTION OF THE LAPLACIAN}

\section{A. Reduced Laplacians}

We have characterized the Hilbert space of the reduced quantum system and studied the geometric structure of the manifold which is underlying the Hilbert space. Now we turn our attention to the Hamiltonian acting on the Hilbert space $L_{2}(M)$. As was anticipated, the Hamiltonian $H$ we treat takes the form $H=\frac{1}{2} \Delta_{M}+V$ with the Laplacian $\Delta_{M}$ and the potential $V$.

First we give a precise definition of the Laplacian. We have been working with the $G$-manifold $M$ equipped with the $G$-invariant measure $\mu_{M}$. We assume that $M$ has no boundary. In what follows, we make another assumption on $M ; M$ is assumed to be oriented and equipped with a Riemannian metric $g_{M}$ which is invariant under the action of $G$. The metric $g_{M}$ induces a volume form $v_{M}$, which is also invariant under the action of $G$. We assume also that $\mu_{M}$ is the measure associated with the volume form $v_{M}$. Let $C_{c}^{\infty}(M)$ be the set of all the $C^{\infty}$ functions on $M$ with compact support. Then the Laplacian $\Delta_{M}: C_{c}^{\infty}(M) \rightarrow C_{c}^{\infty}(M)$ is defined through

$$
\int_{M}\|d f(x)\|_{g_{M}}^{2} v_{M}=\int_{M} f(x)\left(\Delta_{M} f\right)(x) v_{M}
$$

where $\|d f(x)\|_{g_{M}}^{2}$ denotes the norm of $T_{x}^{*} M$ induced by the metric $g_{M}$. We note that the domain of $\Delta_{M}$ is extended in $L_{2}(M)$ to make $\Delta_{M}$ a self-adjoint operator. Of course, in order that this be the case, $M$ has to be assumed to be complete. Since both the metric $g_{M}$ and the volume $v_{M}$ are $G$-invariant, the Laplacian $\Delta_{M}$ is also $G$-invariant; namely, $\Delta_{M}$ commutes with $U(g)$ for any $g$ $\in G$.

Next we turn our attention to the potential energy $V(x)$. It is a smooth function $V: M \rightarrow \boldsymbol{R}$ acting on $f \in L_{2}(M)$ by multiplication as $(V f)(x):=V(x) f(x)$. We assume that $V$ is also $G$-invariant; $V(g x)=V(x)$ for any $g \in G$ and $x \in M$. Thus the action of $V$ also commutes with $U(g)$.

Since each term of the Hamiltonian $H=\frac{1}{2} \Delta_{M}+V$ commutes with $U(g)$ for any $g \in G$, we can apply the decomposition (2.10) and the commutativity (2.24) to both $\Delta_{M}$ and $V$ separately. Hence, $\Delta_{M}$ and $V$ act as $\left(\operatorname{id}_{\mathcal{H} X} \otimes \Delta_{M}\right)$ and $\left(\operatorname{id}_{\mathcal{H} X} \otimes V\right)$ on the reduced Hilbert space $\left(\mathcal{H}^{\chi} \otimes L_{2}(M)\right)^{G}$ $\cong L_{2}\left(M ; \mathcal{H}^{\chi}\right)^{G}$. The Laplacian $\left(\mathrm{id}_{\mathcal{H} \chi} \otimes \Delta_{M}\right)$ with the domain restricted to $\left(\mathcal{H}^{X} \otimes L_{2}(M)\right)^{G}$ $\cong L_{2}\left(M ; \mathcal{H}^{X}\right)^{G}$ is denoted by $\Delta^{\chi}$, and is called a reduced Laplacian. The reduced Laplacian $\Delta^{X}$ is also a self-adjoint operator. Then for an equivariant function $\psi \in C_{c}^{\infty}\left(M ; \mathcal{H}^{X}\right)^{G}$, the defining equation of the Laplacian $\Delta^{\chi}$ takes the form

$$
\int_{M}\|d \psi(x)\|_{g_{M}}^{2} v_{M}=\int_{M}\left\langle\psi(x),\left(\Delta^{\chi} \psi\right)(x)\right\rangle v_{M},
$$

where in the LHS $\|d \psi(x)\|_{g_{M}}^{2}$ denotes the norm of $\mathcal{H}^{\chi} \otimes T_{x}^{*} M$ induced from the metric $g_{M}$.

\section{B. Rotational and vibrational energy operators}

To make further study of $\Delta^{x}$, we make intensive use of the vertical-horizontal decomposition $T_{x} M=V_{x} \oplus H_{x}$ introduced in Sec. III. We have not chosen a specific connection yet. Now we fix the connection by demanding the orthogonality $V_{x} \perp H_{x}$ with respect to the metric $g_{M}(x)$. Then, the $G$-invariance of the metric ensures that $g_{*} H_{x}=H_{g x}$, and hence a unique connection is determined. Since the set of maximum points $M_{\mu}$ is an open dense subset of $M$ as noticed previously and since the connection is smooth when restricted to $M_{\mu}$, the Laplacian on $M_{\mu}$ will be smoothly decomposed into two, vertical and horizontal components, by the use of the connection.

According to the orthogonal decomposition $T_{x}^{*} M=H_{x}^{*} \oplus V_{x}^{*}$, the integrand of the LHS of (4.2) is also written as

$$
\|d \psi(x)\|_{g_{M}}^{2}=\left\|(d \psi(x))_{H}\right\|_{g_{M}}^{2}+\left\|(d \psi(x))_{V}\right\|_{g_{M}}^{2} .
$$


By the definition of the covariant derivative (3.11) and the expression (3.13) in terms of the connection form, the above equation is put in the form

$$
\begin{aligned}
\|d \psi(x)\|_{g_{M}}^{2} & =\left\|\left(d-\rho_{*}^{\chi}\left(\omega_{x}\right)\right) \psi(x)\right\|_{g_{M}}^{2}+\left\|\rho_{*}^{\chi}\left(\omega_{x}\right) \psi(x)\right\|_{g_{M}}^{2} \\
& =\|D \psi(x)\|_{g_{M}}^{2}+\left\|\rho_{*}^{\chi}\left(\omega_{x}\right) \psi(x)\right\|_{g_{M}}^{2}
\end{aligned}
$$

The second term of the RHS of (4.4) proves to be expressed as

$$
\begin{aligned}
\left\|\rho_{*}^{\chi}\left(\omega_{x}\right) \psi(x)\right\|_{g_{M}}^{2} & =g_{M}^{-1}\left\langle\rho_{*}^{\chi}\left(\omega_{x}\right) \psi(x), \rho_{*}^{\chi}\left(\omega_{x}\right) \psi(x)\right\rangle \\
& =-g_{M}^{-1}\left\langle\psi(x), \rho_{*}^{\chi}\left(\omega_{x}\right) \otimes \rho_{*}^{\chi}\left(\omega_{x}\right) \psi(x)\right\rangle \\
& =\left\langle\psi(x), \Lambda_{x}^{\chi} \psi(x)\right\rangle,
\end{aligned}
$$

where we have to make remarks on the notations used; the $g_{M}^{-1}\langle$,$\rangle denotes the inner product on$ $T^{*} M \otimes \mathcal{H}^{\chi}$, the product $\rho_{*}^{\chi}\left(\omega_{x}\right) \otimes \rho_{*}^{\chi}\left(\omega_{x}\right)$ is to be understood as a tensor product in $T^{*} M$ $\otimes T^{*} M \otimes$ End $\mathcal{H}^{X}$ and the $\Lambda_{x}^{X}$ is defined by

$$
\Lambda_{x}^{\chi}:=-g_{M}^{-1}(x) \circ\left(\rho_{*}^{\chi}\left(\omega_{x}\right) \otimes \rho_{*}^{\chi}\left(\omega_{x}\right)\right) \in \operatorname{End} \mathcal{H}^{\chi},
$$

with $g_{M}^{-1}(x)$ taken as the inner product on $T^{*} M \otimes T^{*} M$. We notice also that at the second line of (4.5), we have used the fact that $\left\langle\rho_{*}^{\chi}(\xi) v, v^{\prime}\right\rangle=-\left\langle v, \rho_{*}^{\chi}(\xi) v^{\prime}\right\rangle$ for any $\xi \in \mathbf{g}$ and for any $v, v^{\prime}$ $\in \mathcal{H}^{X}$. The equivariance of the connection form (3.3) and the invariance of the metric are put together to imply that

$$
\Lambda_{g x}^{\chi}=\rho^{\chi}(g) \Lambda_{x}^{\chi} \rho^{\chi}\left(g^{-1}\right) .
$$

Then we observe that the operator $\Lambda^{\chi}$ acting on $L_{2}\left(M ; \mathcal{H}^{\chi}\right)$ through $\left(\Lambda^{\chi} \psi\right)(x):=\Lambda_{x}^{\chi} \psi(x)$ leaves $L_{2}\left(M ; \mathcal{H}^{\chi}\right)^{G}$ invariant, so that one obtains $\Lambda_{x}^{\chi} \in \operatorname{End}\left(\mathcal{H}^{X}\right)^{G_{x}}$. We can put the $\Lambda^{X}$ in another form. Since the inner product $g_{M}(x): T_{x} M \otimes T_{x} M \rightarrow \boldsymbol{R}$ gives rise to an isomorphism $\hat{g}_{M}(x): T_{x} M$ $\rightarrow T_{x}^{*} M$, its inverse $\hat{g}_{M}^{-1}(x): T_{x}^{*} M \rightarrow T_{x} M$ induces an inner product on the cotangent space $g_{M}^{-1}(x): T_{x}^{*} M \otimes T_{x}^{*} M \rightarrow \boldsymbol{R}$ in the dual manner. Hence $g_{M}^{-1}$ is viewed as a symmetric tensor field $g_{M}^{-1}: M \rightarrow T M \otimes T M$. In terms of $g_{M}^{-1}$ along with the connection form $\omega_{x}: T_{x} M \rightarrow \mathbf{g} / \mathbf{g}_{x}$ and the representation of Lie algebra $\rho_{*}^{\chi}: \mathbf{g} \rightarrow$ End $\mathcal{H}^{\chi}$, the tensor field $\Lambda^{\chi}: M \rightarrow$ End $\mathcal{H}^{\chi}$ takes the form,

$$
\Lambda^{\chi}:=-\left(\rho_{*}^{\chi} \otimes \rho_{*}^{\chi}\right) \circ(\omega \otimes \omega) \circ g_{M}^{-1} .
$$

If $\rho^{\chi}$ is not a trivial representation and if $G$ acts on $M$ nontrivially, then $\Lambda^{\chi}$ is a positive definite operator. We call the $\Lambda^{\chi}$ the rotational energy operator, the reason for which comes from molecular mechanics with $G=\mathrm{SO}(3)$. In fact, when applied to molecular mechanics, the quantity (4.5) is interpreted as the rotational energy density.

We proceed to study the first term of the RHS of (4.4). To this end, we have to make a review of Hodge's star operator $*_{M}: \Omega^{k}(M) \rightarrow \Omega^{m-k}(M)$, which is defined through

$$
\alpha \wedge *_{M} \beta=\langle\alpha, \beta\rangle_{g_{M}} v_{M}, \quad \alpha, \beta \in \Omega^{k}(M),
$$

where $\langle\alpha, \beta\rangle$ in the RHS is the inner product on $\wedge^{k} T_{x}^{*} M$ defined by the metric $g_{M}$. Let $\left\{e_{1}, \ldots, e_{m}\right\}$ be a local orthonormal frame field on $M$ with respect to the metric $g_{M}$. Then the star operator $*_{M}$ is explicitly given by

$$
*_{M} \alpha=\frac{1}{k !} \sum_{j_{1}, \cdots j_{k}=1}^{m}\left(i\left(e_{j_{1}}\right) \cdots i\left(e_{j_{k}}\right) \alpha\right)\left(i\left(e_{j_{1}}\right) \ldots i\left(e_{j_{k}}\right) v_{M}\right) .
$$


It is easily verified that $*_{M}{ }_{M} \alpha=(-1)^{k(m-k)} \alpha$. The defining equation (4.1) of the Laplacian $\Delta_{M}$ is then rewritten as

$$
\int_{M}\|d f(x)\|_{g_{M}}^{2} v_{M}=\int_{M} d f \wedge *_{M} d f=\int_{M} d\left(f *_{M} d f\right)-\int_{M} f d *_{M} d f=-\int_{M} f\left(*_{M}^{-1} d *_{M} d f\right) v_{M},
$$

where we have used Stokes' theorem to eliminate the first term on the second line. Then the Laplacian takes the form

$$
\Delta_{M} f=-*_{M}^{-1} d *_{M} d f=-*_{M} d *_{M} d f .
$$

In terms of local coordinates $\left(x^{1}, \ldots, x^{m}\right)$ of $M$, the metric and the volume form are expressed as $g_{M}=\Sigma_{i, j} g_{M i j}(x) d x^{i} \otimes d x^{j}$ and $v_{M}=v_{M}(x) d x^{1} \wedge \cdots \wedge d x^{m}=\sqrt{\operatorname{det} g_{M i j}(x)} d x^{1} \wedge \ldots \wedge d x^{m}$, respectively. The Laplacian $\Delta_{M}$ is then expressed as

$$
\Delta_{M} f=-\frac{1}{v_{M}(x)} \sum_{i, j=1}^{m} \frac{\partial}{\partial x^{i}}\left(v_{M}(x)\left(g_{M}^{-1}\right)^{i j}(x) \frac{\partial f}{\partial x^{j}}\right)
$$

as is well-known.

We examine (4.12) more closely, using the horizontal-vertical decomposition $T_{x} M=H_{x}$ $\oplus V_{x}$. The measure $\mu_{M}$ of $M$ projects to a measure $\mu_{Q}$ of $Q$ through $\pi: M \rightarrow Q$;

$$
\int_{Q} \varphi(q) d \mu_{Q}(q):=\int_{M}\left(\varphi^{\circ} \pi\right)(x) d \mu_{M}(x), \quad \varphi \in C^{0}(Q) .
$$

In what follows, we restrict ourselves to the maximum stratum $M_{\mu}$, which is an open and dense subset of $M$. Let $v_{Q}$ be a volume form on $Q_{\mu}$ associated with the measure $\mu_{Q}$. We can define a Riemannian metric $g_{Q}$ on $Q_{\mu}$ through $\pi^{*} g_{Q}=g_{M} \mid H$, where $g_{M} \mid H$ denote the restriction of the metric $g_{M}: T_{x} M \times T_{x} M \rightarrow \boldsymbol{R}$ to the horizontal subspace; $g_{M} \mid H: H_{x} \times H_{x} \rightarrow \boldsymbol{R}$. Note that the definition of $g_{Q}$ is independent of the choice of $x \in \pi^{-1}(\pi(x))$, because of the $G$-invariance of $g_{M}$. The map $\pi:\left(M_{\mu}, g_{M}\right) \rightarrow\left(Q_{\mu}, g_{Q}\right)$ then becomes a Riemannian submersion. It is to be noted that the volume form $v_{Q}$ does not coincides with the volume form induced from the metric $g_{Q}$. We denote the set of all the horizontal and the vertical $k$-forms on $M_{\mu}$ by

$$
\begin{aligned}
& \Omega_{H}^{k}\left(M_{\mu}\right):=\left\{\alpha \in \Omega^{k}\left(M_{\mu}\right) \mid i(X) \alpha=0, \forall X \in V_{x}, \forall x \in M_{\mu}\right\}, \\
& \Omega_{V}^{k}\left(M_{\mu}\right):=\left\{\alpha \in \Omega^{k}\left(M_{\mu}\right) \mid i(X) \alpha=0, \forall X \in H_{x}, \forall x \in M_{\mu}\right\},
\end{aligned}
$$

respectively. Note that we have already put $\operatorname{dim} M=m, \operatorname{dim} Q_{\mu}=n=m-p$. We define the horizontal and the vertical volume forms, $v_{H} \in \Omega_{H}^{n}\left(M_{\mu}\right)$ and $v_{V} \in \Omega_{V}^{p}\left(M_{\mu}\right)$, through $v_{H}=\pi^{*} v_{Q}$ and $v_{M}=v_{H} \wedge v_{V}$, respectively. It appears that the forms $v_{H}$ and $v_{V}$ are uniquely determined and $G$-invariant.

Moreover, it can be shown that both $v_{H}$ and $v_{V}$ are closed forms. It is easy to verify that $v_{H}$ is closed; $d v_{H}=d\left(\pi^{*} v_{Q}\right)=\pi^{*}\left(d v_{Q}\right)=0$, since $v_{Q}$ is a top form of $Q_{\mu}$. To prove that $d v_{V}=0$, we use a local trivialization over an open set $W \subset Q_{\mu} ; \pi^{-1}(W) \subset M_{\mu}$ is identified with $W \times F$, where $F:=G / G_{\mu}$ is the maximum orbit. The trivialization induces a surjective map $\pi_{F}: \pi^{-1}(W) \rightarrow F$ which is $G$-equivariant, that is, $\pi_{F}(g x)=g \pi_{F}(x)$ for each $g \in G$ and $x$ $\in \pi^{-1}(W)$. A restriction of the map $\pi_{F}$ to each fiber gives a diffeomorphism $\pi^{-1}(q) \cong F$ for each $q \in W$. Let $v_{F}$ be a $G$-invariant volume form on $F$ which is normalized as $\int_{F} v_{F}=1$. Then $v_{F}$ is uniquely determined. Now the definition (4.14) of the measure $\mu_{Q}$ is put in the form, 


$$
\begin{aligned}
\int_{W} \varphi(q) v_{Q} & =\int_{\pi^{-1}(W)}\left(\varphi^{\circ} \pi\right)(x) v_{M} \\
& =\int_{\pi^{-1}(W)}\left(\varphi^{\circ} \pi\right)(x) v_{H} \wedge v_{V} \\
& =\int_{W} \varphi(q) v_{Q}\left(\int_{\pi^{-1}(q)} v_{V}\right) .
\end{aligned}
$$

Since $\varphi$ is arbitrary, this equation implies that the volume form $v_{V}$ restricted to each fiber $\pi^{-1}(q)$ is also normalized as $\int_{\pi^{-1}(q)} v_{V}=1$ for each $q \in Q_{\mu}$, so that $v_{V}$ is a $G$-invariant normalized volume form on each fiber $\pi^{-1}(q)$. It then follows that $v_{V}=\pi_{F}^{*} v_{F}$. As a consequence, one has $d v_{V}=\pi_{F}^{*}\left(d v_{F}\right)=0$, since $v_{F}$ is a top form of $F$.

Using $v_{H}$ and $v_{V}$, we define the horizontal and the vertical star operators $*_{H}: \Omega_{H}^{k}\left(M_{\mu}\right)$ $\rightarrow \Omega_{H}^{n-k}\left(M_{\mu}\right)$ and $*_{V}: \Omega_{V}^{k}\left(M_{\mu}\right) \rightarrow \Omega_{V}^{p-k}\left(M_{\mu}\right)$ through

$$
\begin{gathered}
*_{M} \alpha=\left(*_{H} \alpha\right) \wedge v_{V}, \quad \alpha \in \Omega_{H}^{k}\left(M_{\mu}\right)(k \leqslant n), \\
*_{M} \beta=(-1)^{n} v_{H} \wedge *_{V} \beta, \quad \beta \in \Omega_{V}^{k}\left(M_{\mu}\right)(k \leqslant p),
\end{gathered}
$$

respectively. According to the decomposition $T_{x}^{*} M=H_{x}^{*} \oplus V_{x}^{*}$, we break up $d f$ into $d f=(d f)_{H}$ $+(d f)_{V}$. Then $*_{M} d f$ is accordingly expressed as

$$
*_{M} d f=*_{H}(d f)_{H} \wedge v_{V}+(-1)^{n} v_{H} \wedge *_{V}(d f)_{V} .
$$

Since $d v_{H}=0$ and $d v_{V}=0$, we obtain

$$
d *_{M} d f=\left(d *_{H}(d f)_{H}\right) \wedge v_{V}+v_{H} \wedge\left(d *_{V}(d f)_{V}\right) .
$$

Thus Eq. (4.12) is expressed as

$$
-\Delta_{M} f=*_{M}^{-1} d *_{M} d f=*_{H}^{-1}\left(d *_{H}(d f)_{H}\right)_{H}+*_{V}^{-1}\left(d *_{V}(d f)_{V}\right)_{V},
$$

which means that the Laplacian $\Delta_{M}$ is decomposed into horizontal and vertical components.

The above argument can be extended to $\mathcal{H}^{\chi} \otimes \Omega^{k}\left(M_{\mu}\right) \cong \Omega^{k}\left(M_{\mu} ; \mathcal{H}^{\chi}\right)$ and to $\Omega_{H}^{k}\left(M_{\mu} ; \mathcal{H}^{\chi}\right)^{G}$ straightforwardly; the star operators $*_{M}$ and $*_{H}$ are extended to be applicable to $\mathcal{H}^{X}$-valued forms on $M_{\mu}$ and to $\mathcal{H}^{\chi}$-valued horizontal forms on $M_{\mu}$, respectively. Hence, for an equivariant function $\psi \in C_{c}^{\infty}\left(M_{\mu} ; \mathcal{H}^{\chi}\right)^{G}$, Eq. (4.22) gives rise to

$$
-\Delta^{\chi} \psi=*_{M}^{-1} d *_{M} d \psi=*_{H}^{-1}\left(d *_{H}(d \psi)_{H}\right)_{H}+*_{V}^{-1}\left(d *_{V}(d \psi)_{V}\right)_{V} .
$$

For $\psi \in C_{c}^{\infty}\left(M_{\mu} ; \mathcal{H}^{\chi}\right)^{G}$, we have $(d \psi)_{H}=D \psi$ by the definition of the covariant derivation (3.11). In view of the first term of the RHS of (4.23), we are led to the definition of the adjoint operator $D^{\dagger}: \Omega_{H}^{k+1}\left(M_{\mu} ; \mathcal{H}^{\chi}\right)^{G} \rightarrow \Omega_{H}^{k}\left(M_{\mu} ; \mathcal{H}^{\chi}\right)^{G}$ of $D$;

$$
D^{\dagger}:=-*_{H}^{-1} D *_{H}=-(-1)^{k(n-k)} *_{H} D *_{H} .
$$

By using (4.2), (4.4), (4.5), (4.22), and (4.24), we accomplish the decomposition of the Laplacian $\Delta^{x}$ into the horizontal and the vertical components,

$$
\Delta^{\chi} \psi=D^{\dagger} D \psi+\Lambda^{\chi} \psi, \quad \psi \in C_{c}^{\infty}\left(M_{\mu} ; \mathcal{H}^{\chi}\right)^{G} .
$$

We call the $D^{\dagger} D$ the vibrational energy operator for the reason that the integral (4.2) is interpreted as the vibrational energy when our general formalism is applied to molecular mechanics. 
As was noted above, the smoothness of the horizontal-vertical decomposition is ensured only in the open dense subset $M_{\mu} \subset M$, so that the RHS of (4.25) makes sense only in $M_{\mu}$. However, $\Delta^{\chi}$ is actually a self-adjoint operator on $L_{2}\left(M ; \mathcal{H}^{\chi}\right)$ by definition, so that the LHS of (4.2) holds for $\psi$ defined throughout $M$. Hence we may expect that some boundary condition arises on $\partial M_{\mu}=M_{\text {sing }}$ to make $D^{\dagger} D+\Lambda^{\chi}$ into a self-adjoint operator on $L_{2}\left(M ; \mathcal{H}^{\chi}\right)$. Since the requirement imposed on $\psi$ is that $\psi$ is to be equivariant, we obtain the boundary condition on $\partial M_{\mu}$,

$$
\rho^{\chi}(g) \psi(x)=\psi(x), \quad g \in G_{x}, \quad x \in \partial M_{\mu},
$$

or, in the form of derivative,

$$
\rho_{*}^{\chi}(\xi) \psi(x)=0, \quad \xi \in \mathbf{g}_{x}, \quad x \in \partial M_{\mu}
$$

In case of $G_{x} \neq\{e\}$ for the maximum orbit type $\mu$, the equivariant functions are, of course, subject to the condition $\rho^{\chi}(g) \psi(x)=\psi(x)$ for $g \in G_{x}, x \in M_{\mu}$. The condition (4.26) or (4.27) states that at singular points the equivariant functions are subject to a stronger condition since $\operatorname{dim} G_{x}$ rises up at singular points.

\section{Angular momentum and inertia tensor}

Now we wish to introduce the angular momentum and the inertia tensor, which are closely related with the connection form and the rotational energy operator $\Lambda^{\chi}$.

The angular momentum is a map $L: T^{*} M \rightarrow \mathbf{g}^{*}$; which is defined through

$$
\left\langle L_{x}(p), \xi\right\rangle:=\left\langle p, \theta_{x}(\xi)\right\rangle, \quad p \in T_{x}^{*} M, \xi \in \mathbf{g},
$$

where $\langle\cdot, \cdot\rangle$ 's in the LHS and in the RHS denote the pairing between $\mathbf{g}^{*}$ and $\mathbf{g}$ and that between $T_{x}^{*} M$ and $T_{x} M$, respectively, and $\theta_{x}(\xi)\left(=\xi_{M}(x)\right)$ is the infinitesimal generator induced by $\xi$ $\in \mathbf{g}$. The angular momentum $L: T^{*} M \rightarrow \mathbf{g}^{*}$ is a typical example of momentum maps due to Marsden and Souriau. ${ }^{26}$ By the use of the isomorphism $\hat{g}_{M}: T M \rightarrow T^{*} M$, the angular momentum can be rewritten as the map $\hat{L}:=L \circ \hat{g}_{M}: T M \rightarrow \mathbf{g}^{*} ; v \mapsto \hat{L}_{x}(v)$, which is expressed as

$$
\left\langle\hat{L}_{x}(v), \xi\right\rangle:=g_{M}\left(v, \theta_{x}(\xi)\right), \quad v \in T_{x} M, \xi \in \mathbf{g} .
$$

Namely, $\hat{L}$ is a $\mathbf{g}^{*}$-valued one-form on $M$, which we call the angular momentum form.

The inertia tensor is a tensor field $I: M \rightarrow \mathbf{g}^{*} \otimes \mathbf{g}^{*} ; x \mapsto I_{x}$, which is defined through

$$
I_{x}(\xi, \zeta):=g_{M}\left(\theta_{x}(\xi), \theta_{x}(\zeta)\right), \xi, \zeta \in \mathbf{g} .
$$

On account of $\theta_{g x}\left(\operatorname{Ad}_{g} \xi\right)=g_{*}\left(\theta_{x}(\xi)\right)$ and of $g^{*} g_{M}=g_{M}$ for any $g \in G$, the inertia tensor transforms according to

$$
I_{g x}\left(\operatorname{Ad}_{g} \xi, \operatorname{Ad}_{g} \zeta\right)=I_{x}(\xi, \zeta)
$$

In other words, the map $I: M \rightarrow \mathbf{g}^{*} \otimes \mathbf{g}^{*}$ is equivariant; $g^{*} I=\left(\mathrm{Ad}_{g^{-1}}^{*} \otimes \mathrm{Ad}_{g^{-1}}^{*}\right) I$. For an arbitrary $x \in M$ fixed, the quadratic form $I_{x}: \mathbf{g} \otimes \mathbf{g} \rightarrow \boldsymbol{R}$ can be regarded as a map $\hat{I}_{x}: \mathbf{g} \rightarrow \mathbf{g}^{*}$, which has $\operatorname{Ker} \hat{I}_{x}=\mathbf{g}_{x}$ and $\operatorname{Im} \hat{I}_{x}=\left\{\phi \in \mathbf{g}^{*} \mid\langle\phi, \xi\rangle=0, \forall \xi \in \mathbf{g}_{x}\right\} \cong\left(\mathbf{g} / \mathbf{g}_{x}\right)^{*}$. Then it can give rise to an isomorphism $\widetilde{I}_{x}: \mathbf{g} / \mathbf{g}_{x} \stackrel{\sim}{\rightarrow}\left(\mathbf{g} / \mathbf{g}_{x}\right)^{*}$. Hence, there exists the inverse $\left(\widetilde{I}_{x}\right)^{-1}:\left(\mathbf{g} / \mathbf{g}_{x}\right)^{*} \stackrel{\sim}{\rightarrow} \mathbf{g} / \mathbf{g}_{x}$, which is identified with a quadratic form $\left(\widetilde{I}_{x}\right)^{-1}:\left(\mathbf{g} / \mathbf{g}_{x}\right)^{*} \otimes\left(\mathbf{g} / \mathbf{g}_{x}\right)^{*} \rightarrow \boldsymbol{R}$. The map $\hat{I}_{x}$ will be referred to as an inertia operator. The inertia operator is called the locked inertia tensor by Simo et al. ${ }^{27}$ The inertia operator was first introduced by Guichardet, ${ }^{3}$ and used in Refs. 8,7 to break up the total energy into the sum of rotational and vibrational energies.

From definition, the angular momentum, the inertia tensor, and the connection form turn out to be related by 


$$
\hat{L}=\hat{I} \circ \omega
$$

where each symbol is to be understood as follows: $\hat{L}_{x}: T_{x} M \rightarrow \mathbf{g}^{*}, \omega_{x}: T_{x} M \rightarrow \mathbf{g} / \mathbf{g}_{x}$, and $\hat{I}_{x}: \mathbf{g} / \mathbf{g}_{x}$ $\rightarrow \mathbf{g}^{*}$. A proof of (4.32) runs as follows: First, the identity

$$
\hat{L} \circ \theta=\hat{I},
$$

can be proved by a straightforward calculation. In fact, for any $\xi, \zeta \in \mathbf{g}$, we have

$$
\left\langle\left(\hat{L}^{\circ} \theta_{x}\right)(\xi), \zeta\right\rangle=g_{M}\left(\theta_{x}(\xi), \theta_{x}(\zeta)\right)=I_{x}(\xi, \zeta)=\left\langle\hat{I}_{x}(\xi), \zeta\right\rangle .
$$

Next, from the identity (4.33) it is deduced that

$$
\hat{L} \circ \theta \circ \omega=\hat{I} \circ \omega .
$$

From the definition of the angular momentum form (4.29), it can be shown that $\operatorname{Ker} \hat{L}_{x}=H_{x}$, so that Eq. (4.32) holds on $H_{x}$. Moreover, since $\widetilde{\theta}_{x}^{\circ} \omega_{x}: T_{x} M=V_{x} \oplus H_{x} \rightarrow V_{x}$ is a projection as was noted at (3.4), Eq. (4.35) shows that (4.32) holds on $V_{x}$. The proof is thus completed. Since $\operatorname{Im} \hat{L}_{x} \cong\left(\mathbf{g} / \mathbf{g}_{x}\right)^{*}$, we may rewrite (4.32) as $\hat{L}=\widetilde{I} \circ \omega$, so that the connection form is put in the form

$$
\omega=\widetilde{I}^{-1} \circ \hat{L} .
$$

This formula will be used to write out the connection form $\omega$ in molecular mechanics.

Owing to the definition of the inertia tensor, $I_{x}=g_{M}{ }^{\circ}\left(\theta_{x} \otimes \theta_{x}\right)$, the metric $g_{M} \mid V$ restricted to the vertical subspace takes the form,

$$
g_{M} \mid V=I^{\circ}(\omega \otimes \omega)=\langle\hat{L}, \omega\rangle,
$$

where use has been made of $\widetilde{\theta}_{x}^{\circ} \omega_{x} \mid V_{x}=\mathrm{id} V_{x}$, another expression of (3.4). Equation (4.37) can be looked upon as describing the rotational energy in classical mechanics. Since the reduced quadratic form $\widetilde{I}_{x}=g_{M^{\circ}}\left(\widetilde{\theta}_{x} \otimes \widetilde{\theta}_{x}\right): \mathbf{g} / \mathbf{g}_{x} \otimes \mathbf{g} / \mathbf{g}_{x} \rightarrow \boldsymbol{R}$ is nondegenerate, it has the inverse quadratic form $\left(\widetilde{I}_{x}\right)^{-1}=g_{M}^{-1} \circ\left(\omega_{x}^{*} \otimes \omega_{x}^{*}\right):\left(\mathbf{g} / \mathbf{g}_{x}\right)^{*} \otimes\left(\mathbf{g} / \mathbf{g}_{x}\right)^{*} \rightarrow \boldsymbol{R}$, which is expressed as a tensor field, $x \mapsto\left(\widetilde{I}_{x}\right)^{-1}$ $\in \mathbf{g} / \mathbf{g}_{x} \otimes \mathbf{g} / \mathbf{g}_{x}$,

$$
\left(\widetilde{I}_{x}\right)^{-1}=\left(\omega_{x} \otimes \omega_{x}\right) \circ g_{M}^{-1},
$$

where $g_{M}^{-1}$ is regarded as a symmetric tensor field $M \rightarrow T M \otimes T M$. From (4.8) and (4.38), we obtain a formula to express $\Lambda^{\chi}$, in terms of the inertia tensor, as

$$
\Lambda^{\chi}=-\left(\rho_{*}^{\chi} \otimes \rho_{*}^{\chi}\right) \circ \widetilde{I}^{-1}
$$

\section{Coordinate representation}

Now we wish to provide a coordinate representation of the reduced Laplacian given in (4.25). Take local coordinates $\left(q^{1}, q^{2}, \ldots, q^{n}\right)$ on an open subset $W$ of $Q_{\mu}$. Then the metric $g_{Q}$ and the volume form $v_{Q}$ take the form $g_{Q}=\Sigma_{i, j} g_{Q i j}(q) d q^{i} \otimes d q^{j}$ and $v_{Q}=v_{Q}(q) d q^{1} \wedge \cdots \wedge d q^{n}$, respectively. For each $x \in \pi^{-1}(W) \subset M_{\mu}$, we take a basis $\left\{\xi_{1}(x), \ldots, \xi_{p}(x)\right\}$ of $\mathbf{g} / \mathbf{g}_{x}$, where we have used the same notation for elements of $\mathbf{g} / \mathbf{g}_{x}$ as those for $\mathbf{g}$ for simplicity. Then the components of the reduced inertia tensor $\widetilde{I}_{x}$ are defined by

$$
\left(\widetilde{I}_{x}\right)_{\alpha \beta}:=\widetilde{I}_{x}\left(\xi_{\alpha}(x), \xi_{\beta}(x)\right), \quad \alpha, \beta=1, \ldots, p=\operatorname{dim} \mathbf{g} / \mathbf{g}_{x}
$$


which gives a symmetric positive definite matrix of rank $p$. The components of its inverse are denoted by $\left(\widetilde{I}_{x}^{-1}\right)^{\alpha \beta}$. Let $\sigma$ be a local section on $W$ of the bundle $\pi: M_{\mu} \rightarrow Q_{\mu}$, that is, a differentiable map $\sigma: W \rightarrow M_{\mu}$ such that $\pi^{\circ} \sigma=\mathrm{id}_{W}$. Then an equivariant function $\psi \in C_{c}^{\infty}\left(M ; \mathcal{H}^{X}\right)^{G}$ is pulled back to a function $\sigma^{*} \psi: W \rightarrow \mathcal{H}^{\chi}$ of the coordinates $\left(q^{1}, q^{2}, \ldots, q^{n}\right)$. Our aim is to obtain a coordinate expression of $\sigma^{*} \Delta^{\chi} \psi$, according to the decomposition $\Delta^{\chi}=D^{\dagger} D+\Lambda^{\chi}$. The covariant derivative (3.13) with $\alpha=\psi$ is expressed, in terms of $\left(q^{1}, q^{2}, \ldots, q^{n}\right)$, as

$$
\left(\sigma^{*} D \psi\right)(q)=\sum_{i=1}^{n}\left(\frac{\partial}{\partial q^{i}}-\left(\rho_{*}^{\chi} \circ \omega^{\circ} \sigma_{*}\right)\left(\frac{\partial}{\partial q^{i}}\right)\right)\left(\sigma^{*} \psi\right)(q) d q^{i} .
$$

It then turns out from (4.24), (4.25), and (4.39) that

$$
\begin{aligned}
\left(\sigma^{*} \Delta^{\chi} \psi\right)(q)= & -\frac{1}{v_{Q}(q)} \sum_{i, j=1}^{n}\left(\frac{\partial}{\partial q^{i}}-\left(\rho_{*}^{\chi} \circ \omega^{\circ} \sigma_{*}\right)\left(\frac{\partial}{\partial q^{i}}\right)\right) v_{Q}(q)\left(g_{Q}^{-1}\right)^{i j}(q) \\
& \times\left(\frac{\partial}{\partial q^{j}}-\left(\rho_{*}^{\chi \circ} \omega^{\circ} \sigma_{*}\right)\left(\frac{\partial}{\partial q^{j}}\right)\right)\left(\sigma^{*} \psi\right)(q) \\
& -\sum_{\alpha, \beta=1}^{p}\left(\widetilde{I}_{\sigma(q)}^{-1}\right)^{\alpha \beta}\left(\rho_{*}^{\chi} \circ \xi_{\alpha^{\circ}} \sigma\right)(q)\left(\rho_{*}^{\chi} \circ \xi_{\beta^{\circ}} \sigma\right)(q)\left(\sigma^{*} \psi\right)(q) .
\end{aligned}
$$

It should be noted here that $\omega\left(\sigma_{*}\left(\partial / \partial q^{i}\right)\right)$ and $\rho_{*}^{\chi}\left(\omega\left(\sigma_{*}\left(\partial / \partial q^{i}\right)\right)\right)$ denote a component of a "gauge potential" and its representation as a matrix acting on $\mathcal{H}^{X}$, respectively. Further, $\rho_{*}^{\chi}\left(\xi_{\alpha}(\sigma(q))\right)$ denote a matrix representation of the infinitesimal generator (or the angular momentum operator) induced by $\xi_{\alpha}(x) \in \mathbf{g}$. Equation (4.42) is one of our main results.

If there exists a global section $\sigma: Q_{\mu} \rightarrow M_{\mu}$, the fiber bundle $\pi: M_{\mu} \rightarrow Q_{\mu}$ becomes a trivial bundle; $M_{\mu} \cong Q_{\mu} \times\left(G / G_{\mu}\right)$, and $\sigma^{*} \psi$ becomes a smooth function over the entire domain $Q_{\mu}$. In this case, the procedure of reduction is nothing but separation of variables. The reduction method is a generalization of the method of separation of variables.

Equation (4.42) makes sense only in the maximum component, $Q_{\mu}$, of the orbit space $Q$. At a singular point $q \in \partial Q_{\mu}$, the rank of the inertia tensor $I_{\sigma(q)}$ decreases abruptly. As was noticed earlier, the equivariance condition provides the boundary condition imposed on $\psi$ and hence on $\sigma^{*} \psi$, which is put in the form

$$
\rho_{*}^{\chi}(\xi)\left(\sigma^{*} \psi\right)(q)=0, \quad \xi \in \mathbf{g}_{\sigma(q)} .
$$

If $\mathbf{g}_{\sigma(q)}=\{0\}$ in $M_{\mu}$, this imposes no condition on $\sigma^{*} \psi$. At singular points $q \in \partial Q_{\mu}$, the dimension of the isotropy algebra $\mathbf{g}_{\sigma(q)}$ jumps up, so that the value of $\sigma^{*} \psi$ is more strongly restricted there. The operator (4.42) should be accompanied by the condition (4.43). This is another one of our main results.

\section{E. Example: Quantum mechanics on a plane}

Quantum mechanics in a two-dimensional Euclidean space provides a simple but nontrivial example of the formulation constructed above.

First we take $M=\boldsymbol{R}^{2}$ and $G=\mathrm{SO}(2)$. Then $\mathrm{SO}(2)$ acts on $\boldsymbol{R}^{2}$ in the usual manner. Let $(r, \phi)$ be the polar coordinate of $\boldsymbol{R}^{2}$. Then the orbit space becomes $Q=\boldsymbol{R}_{\geqslant 0}$, which is nothing but the radius coordinate $r \geqslant 0$. A point with $r \neq 0$ has a maximum orbit $S^{1}$. The origin $r=0$ has a singular orbit, that consists of a single point $\{0\}$. In this example we have $M_{\mu}=\boldsymbol{R}^{2}-\{0\}, \partial M_{\mu}=\{0\}, Q_{\mu}$ $=\boldsymbol{R}_{>0}$, and $\partial Q_{\mu}=\{0\}$. Since $\boldsymbol{R}^{2}-\{0\} \cong \boldsymbol{R}_{>0} \times S^{1}$, the fiber bundle $\pi: M_{\mu} \rightarrow Q_{\mu}$ is trivial. Then the reduction method becomes the method of separation of variables in this case.

The space $\boldsymbol{R}^{2}$ is equipped with the standard metric $g_{M}=d r^{2}+r^{2} d \phi^{2}$ and the standard measure $d \mu_{M}=r d r d \phi$. Then the projected measure of $\boldsymbol{R}_{\geqslant 0}$ is $d \mu_{Q}=2 \pi r d r$. At each maximum point $x$ $\in \boldsymbol{R}^{2}$ with $r \neq 0$, the vertical and horizontal subspaces are given by 


$$
V_{x}=\boldsymbol{R} \frac{\partial}{\partial \phi}, \quad H_{x}=\boldsymbol{R} \frac{\partial}{\partial r},
$$

respectively. At the singular point $r=0$, one has $V_{0}=\{0\}$ and $H_{0} \cong \boldsymbol{R}^{2}$. The metric on $\boldsymbol{R}_{>0}$ is $g_{Q}=d r^{2}$, and thereby one obtains the Riemannian submersion $\pi: M_{\mu} \rightarrow Q_{\mu}$. It should be noted that $d \mu_{Q}=2 \pi r d r$ does not coincide with the metric volume $d r$.

Any irreducible unitary representation of $\mathrm{SO}(2)$ is one-dimensional. It is labeled by an integer $n \in \boldsymbol{Z}$ and defined by

$$
\rho_{n}: \operatorname{SO}(2) \rightarrow U(1) ; \quad\left(\begin{array}{rr}
\cos \alpha & -\sin \alpha \\
\sin \alpha & \cos \alpha
\end{array}\right) \mapsto e^{i n \alpha}
$$

Accordingly, an equivariant function $\psi: \boldsymbol{R}^{2} \rightarrow \boldsymbol{C}$ which satisfies $\psi(g x)=\rho_{n}(g) \psi(x)$ becomes a function $\psi_{n}(r, \phi)$ subject to the condition $\psi_{n}(r, \phi+\alpha)=e^{i n \alpha} \psi_{n}(r, \phi)$. Thus we can put $\psi_{n}$ in the form $\psi_{n}(r, \phi)=e^{i n \phi} f_{n}(r)$. Here the decomposition (2.10) with the projection operators (2.7) realizes the ordinary Fourier expansion in angular coordinate, $\psi(r, \theta)=\Sigma_{n=-\infty}^{\infty} e^{i n \phi} f_{n}(r)$. Since $\mathrm{SO}(2)$ is an isotropy group at the origin $r=0$, smooth equivariant functions $\psi_{n}$ must satisfy

$$
\left.\frac{\partial}{\partial \phi} \psi_{n}\right|_{r=0}=0
$$

which has an alternative expression

$$
n f_{n}(0)=0
$$

This boundary condition illustrates the general condition (4.43).

We proceed to reduce the ordinary Laplacian. The metric on the cotangent bundle $T^{*} \boldsymbol{R}^{2}$ is expressed as

$$
g_{M}^{-1}=\frac{\partial}{\partial r} \otimes \frac{\partial}{\partial r}+\frac{1}{r^{2}} \frac{\partial}{\partial \phi} \otimes \frac{\partial}{\partial \phi} .
$$

To obtain the reduced Laplacian, we calculate the integral (4.2) for $\psi_{n}$,

$$
\begin{aligned}
\int_{R^{2}}\left\|d \psi_{n}\right\|_{g_{M}}^{2} d \mu_{M} & =\int_{R^{2}}\left(\left|\frac{\partial \psi_{n}}{\partial r}\right|^{2}+\frac{1}{r^{2}}\left|\frac{\partial \psi_{n}}{\partial \phi}\right|^{2}\right) r d r d \phi=\int_{0}^{\infty}\left(\left|\frac{d f_{n}}{d r}\right|^{2}+\frac{n^{2}}{r^{2}}\left|f_{n}\right|^{2}\right) 2 \pi r d r \\
& =\left[2 \pi r \overline{f_{n}} \frac{d f_{n}}{d r}\right]_{0}^{\infty}+\int_{0}^{\infty} \overline{f_{n}}\left(-\frac{1}{r} \frac{d}{d r} r \frac{d f_{n}}{d r}+\frac{n^{2}}{r^{2}} f_{n}\right) 2 \pi r d r .
\end{aligned}
$$

Since $f_{n}(r)$ is bounded as $r \rightarrow 0$ from (4.47), and since $r\left(d f_{n} / d r\right) \rightarrow 0$ as $r \rightarrow 0$ from the RHS of the first line, the boundary term at $r=0$ vanishes. The other term at $r=\infty$ vanishes because of the assumption that $\psi_{n}$ has a compact support. Thus we are left with the reduced Laplacian,

$$
\Delta_{n} f_{n}=-\frac{1}{r} \frac{d}{d r} r \frac{d f_{n}}{d r}+\frac{n^{2}}{r^{2}} f_{n}
$$

We note here that the boundary condition (4.47) says that $f_{n}(0)=0$ for $n \neq 0$ and that $f_{0}(0)$ is bounded for $n=0$. This result gives an example of the general formula (4.42). An eigenfunction of the Laplacian $\Delta_{n}$ with an eigenvalue $E>0$ is the $n$th Bessel function $J_{n}(\sqrt{E} r)$. The Neumann function $Y_{n}(\sqrt{E} r)$ does not satisfy the boundary condition (4.47). 


\section{F. Rigid body}

We have to notice here that our theory covers quantum mechanics of rigid bodies. In mechanics, a rigid body is defined as a collection of mass points in three dimensions, in which their mutual distances are kept fixed. In this case, the symmetry group $G$ is $\mathrm{SO}(3)$ and the configuration space $M$ becomes a single $G$-orbit. Hence the orbit space $Q$ reduces to a single point. When the orbit is maximal, $M$ becomes $\mathrm{SO}(3)$ and the inertia tensor $I$ is nondegenerated. When the orbit is singular, $M$ is isomorphic to $S^{2}$ or a single point, and the inertia tensor is of rank two or zero, respectively. For a rigid body, the horizontal component of the Laplacian (4.25), or the vibrational energy operator, vanishes, and therefore the Laplacian reduces to the rotational energy operator,

$$
\Delta^{\chi}=\Lambda^{\chi}=-\left(\rho_{*}^{\chi} \otimes \rho_{*}^{\chi}\right) \widetilde{I}^{-1},
$$

which is the Casimir operator acting on the representation space $\mathcal{H}^{x}$ up to a normalization constant. In the language of physics, since the rigid body executes no vibrational motion, it has only rotational energy, which is determined by the angular momentum. A simple example will be given in Sec. V.

\section{QUANTUM MOLECULAR MECHANICS}

\section{A. Jacobi vectors}

In the previous sections, we have set up a general formulation for reduction of quantum dynamical systems on the configuration space $M$ with symmetry $G$. The Hilbert space $L_{2}(M)$ is decomposed into the orthogonal direct sum of the spaces of equivariant functions according to the irreducible unitary representations of $G$, as was shown in (2.35). The Laplacian $\Delta_{M}$ is accordingly reduced to the operator $\Delta^{X}$ of (4.25) acting on each space of equivariant functions $L_{2}\left(M ; \mathcal{H}^{\chi}\right)^{G}$. We have studied quantum mechanics on $M=\boldsymbol{R}^{2}$ with symmetry $G=\mathrm{SO}(2)$ to give a concrete example. It was a well-known but nontrivial example in which reduction by symmetry serves as the method of separating variables.

Here, we wish to apply the general formulation to molecular mechanics, which is the original problem that motivates us. We consider a molecular system consisting of $N$ atoms in $\boldsymbol{R}^{3}$. The configuration of the molecule is described as an ennuple $\left(\boldsymbol{x}_{1}, \ldots, \boldsymbol{x}_{N}\right)$ of the position, $\boldsymbol{x}_{i} \in \boldsymbol{R}^{3}$, of each atom. Masses of the atoms are denoted by $\left(m_{1}, \ldots, m_{N}\right)$ with $m_{i} \in \boldsymbol{R}_{>0}$. Assume that we are working with the center-of-mass system,

$$
M=\left\{\left(\boldsymbol{x}_{1}, \ldots, \boldsymbol{x}_{N}\right) \in\left(\boldsymbol{R}^{3}\right)^{N} \mid \sum_{i=1}^{N} m_{i} \boldsymbol{x}_{i}=0\right\},
$$

which is a linear subspace of $\left(\boldsymbol{R}^{3}\right)^{N}$. Let $g \in G=\mathrm{SO}(3)$ act on $x=\left(x_{1}, \ldots, \boldsymbol{x}_{N}\right) \in M$ by $g x$ $=\left(g \boldsymbol{x}_{1}, \ldots, g \boldsymbol{x}_{N}\right)$. We call $M$ and $Q=M / \mathrm{SO}(3)$ the molecular configuration space and the shape space, respectively. We may regard $x=\left(\boldsymbol{x}_{1}, \ldots, \boldsymbol{x}_{N}\right)$ as a $3 \times N$ matrix. According as the rank of $x$ is $3,2,1$, or 0 , the configuration $x$ is called a generic configuration, a planar one, a collinear one, or a collision one, respectively. A generic or planar configuration has a maximum orbit which is diffeomorphic to $\mathrm{SO}(3)$. A collinear configuration, in which all the atoms are placed along a line, has a singular orbit which is diffeomorphic to $S^{2}$. A collision configuration $x=(0, \ldots, 0)$ has another singular orbit which consists of a single point. We are going to review a geometric setting on $M$ in what follows. The topology of the shape space $Q$ will be studied in the next subsection.

While a tangent vector $v \in T_{x} M$ is denoted by $v=\left(\boldsymbol{v}_{1}, \ldots, \boldsymbol{v}_{N}\right) \in\left(\boldsymbol{R}^{3}\right)^{N}$ along with the condition $\Sigma_{i=1}^{N} m_{i} \boldsymbol{v}_{i}=0$, a cotangent vector $p \in T_{x}^{*} M$ is denoted by $p=\left(\boldsymbol{p}_{1}, \ldots, \boldsymbol{p}_{N}\right) \in\left(\boldsymbol{R}^{3}\right)^{N}$ along with the condition $\sum_{i=1}^{N} \boldsymbol{p}_{i}=0$. The pairing between $T_{x} M$ and $T_{x}^{*} M$ is given by $\langle p, v\rangle:=\sum_{i=1}^{N}\left(\boldsymbol{p}_{i}, \boldsymbol{v}_{i}\right)$, where $(\cdot, \cdot)$ denotes the standard inner product of $\boldsymbol{R}^{3}$. Each element of the Lie algebra $\xi \in \mathbf{s o}(3)$ is identified with a vector $\boldsymbol{\xi} \in \boldsymbol{R}^{3}$ and induces the infinitesimal transformation $\theta_{x}(\boldsymbol{\xi})=\left(\boldsymbol{\xi} \times \boldsymbol{x}_{1}, \ldots, \boldsymbol{\xi} \times \boldsymbol{x}_{N}\right)$, where $\times$ means the standard vector product in $\boldsymbol{R}^{3}$. Moreover, the dual space of the Lie algebra so(3)* is also identified with $\boldsymbol{R}^{3}$. 
The space $\left(\boldsymbol{R}^{3}\right)^{N}$ is equipped with a Riemannian metric $K$,

$$
K:=\sum_{i=1}^{N} m_{i}\left(d \boldsymbol{x}_{i}, d \boldsymbol{x}_{i}\right)
$$

Although $K$ is twice the kinetic energy, we will call it the kinetic energy simply. The subspace $M \subset\left(\boldsymbol{R}^{3}\right)^{N}$ inherits the Riemannian metric $K$. From the definition (4.28), the angular momentum $\boldsymbol{L}: T^{*} M \rightarrow \mathbf{s o}(3)^{*} \cong \boldsymbol{R}^{3}$ is expressed as

$$
\left(\boldsymbol{L}_{x}(p), \boldsymbol{\xi}\right)=\left\langle p, \theta_{x}(\boldsymbol{\xi})\right\rangle,=\sum_{i=1}^{N}\left(\boldsymbol{p}_{i}, \boldsymbol{\xi} \times \boldsymbol{x}_{i}\right)=\left(\sum_{i=1}^{N} \boldsymbol{x}_{i} \times \boldsymbol{p}_{i}, \boldsymbol{\xi}\right),
$$

and hence takes the usual form

$$
\boldsymbol{L}=\sum_{i=1}^{N} \boldsymbol{x}_{i} \times \boldsymbol{p}_{i}
$$

According to (4.29), the angular momentum form $\hat{\boldsymbol{L}}: T M \rightarrow \mathbf{s o}(3) *$ is expressed as

$$
\left(\hat{\boldsymbol{L}}_{x}(v), \boldsymbol{\xi}\right)=K\left(v, \theta_{x}(\boldsymbol{\xi})\right)=\sum_{i=1}^{N} m_{i}\left(\boldsymbol{v}_{i}, \boldsymbol{\xi} \times \boldsymbol{x}_{i}\right)=\left(\sum_{i=1}^{N} m_{i} \boldsymbol{x}_{i} \times \boldsymbol{v}_{i}, \boldsymbol{\xi}\right),
$$

which implies that

$$
\hat{\boldsymbol{L}}=\sum_{i=1}^{N} m_{i} \boldsymbol{x}_{i} \times d \boldsymbol{x}_{i}
$$

The Jacobi vectors are of great help in describing many-body systems. Let us remind us of the definition of the Jacobi vectors. By $M_{i} \in \boldsymbol{R}_{>0}$ and $\boldsymbol{X}_{i} \in \boldsymbol{R}^{3}$ we denote the sum of the masses from the first to the $i$ th atom and the center-of-mass of the set of $i$ atoms, respectively,

$$
M_{i}:=\sum_{j=1}^{i} m_{j}, \quad \boldsymbol{X}_{i}:=\frac{1}{M_{i}} \sum_{j=1}^{i} m_{j} \boldsymbol{x}_{j} \quad(i=1, \ldots, N) .
$$

In particular, one has $\boldsymbol{X}_{1}=\boldsymbol{x}_{1}$, and $\boldsymbol{X}_{N}$ is equal to the center of mass of the whole system. Then the Jacobi vectors $\left(\boldsymbol{r}_{0}^{(N)}, \boldsymbol{r}_{1}^{(N)}, \ldots, \boldsymbol{r}_{N-1}^{(N)}\right)$ are defined by

$$
\boldsymbol{r}_{0}^{(N)}:=\sqrt{M_{N}} \boldsymbol{X}_{N}, \quad \boldsymbol{r}_{i}^{(N)}:=\left(\frac{1}{M_{i}}+\frac{1}{m_{i+1}}\right)^{-(1 / 2)}\left(\boldsymbol{x}_{i+1}-\boldsymbol{X}_{i}\right) \quad(i=1, \ldots, N-1) .
$$

Of course, in the center-of-mass system, one has $\boldsymbol{X}_{N}=\boldsymbol{r}_{0}^{(N)}=0$. The Jacobi vectors $\left(\boldsymbol{r}_{1}^{(N)}, \ldots, \boldsymbol{r}_{N-1}^{(N)}\right)$ provide a coordinate system to $M$, and give rise to the isomorphism $M$ $\cong\left(\boldsymbol{R}^{3}\right)^{(N-1)}$.

Good use is made of the Jacobi vectors to prove the additivity of the kinetic energy $K$ and of the angular momentum $\hat{\boldsymbol{L}}$ in the number of atoms. In fact, one can verify that

$$
\begin{gathered}
K^{(N)}:=\sum_{i=1}^{N} m_{i}\left(d \boldsymbol{x}_{i}, d \boldsymbol{x}_{i}\right)=\sum_{i=0}^{N-1}\left(d \boldsymbol{r}_{i}^{(N)}, d \boldsymbol{r}_{i}^{(N)}\right), \\
\hat{\boldsymbol{L}}^{(N)}:=\sum_{i=1}^{N} m_{i} \boldsymbol{x}_{i} \times d \boldsymbol{x}_{i}=\sum_{i=0}^{N-1} \boldsymbol{r}_{i}^{(N)} \times d \boldsymbol{r}_{i}^{(N)} .
\end{gathered}
$$


The additivity of $K^{(N)}$ can be proved by induction with respect to $N$. A straightforward calculation yields

$$
\begin{aligned}
K^{(N+1)}-K^{(N)} & =\left\|d \boldsymbol{r}_{0}^{(N+1)}\right\|^{2}+\left\|d \boldsymbol{r}_{N}^{(N+1)}\right\|^{2}-\left\|d \boldsymbol{r}_{0}^{(N)}\right\|^{2} \\
& =M_{N+1}\left\|d \boldsymbol{X}_{N+1}\right\|\left\|^{2}+\frac{M_{N} m_{N+1}}{M_{N}+m_{N+1}}\right\| d \boldsymbol{x}_{N+1}-d \boldsymbol{X}_{N}\left\|^{2}-M_{N}\right\| d \boldsymbol{X}_{N} \|^{2} \\
& =\frac{1}{M_{N+1}}\left\|M_{N} d \boldsymbol{X}_{N}+m_{N+1} d \boldsymbol{x}_{N+1}\right\|^{2}+\frac{M_{N} m_{N+1}}{M_{N+1}}\left\|d \boldsymbol{x}_{N+1}-d \boldsymbol{X}_{N}\right\|^{2}-M_{N}\left\|d \boldsymbol{X}_{N}\right\|^{2} \\
& =\frac{M_{N}^{2}+M_{N} m_{N+1}-M_{N} M_{N+1}}{M_{N+1}}\left\|d \boldsymbol{X}_{N}\right\|^{2}+\frac{m_{N+1}^{2}+M_{N} m_{N+1}}{M_{N+1}}\left\|d \boldsymbol{x}_{N+1}\right\|^{2} \\
& =m_{N+1}\left\|d \boldsymbol{x}_{N+1}\right\|^{2} .
\end{aligned}
$$

In a similar manner, the additivity of the angular momentum is verified as follows:

$$
\begin{aligned}
\hat{\boldsymbol{L}}^{(N+1)}-\hat{\boldsymbol{L}}^{(N)}= & \boldsymbol{r}_{0}^{(N+1)} \times d \boldsymbol{r}_{0}^{(N+1)}+\boldsymbol{r}_{N}^{(N+1)} \times d \boldsymbol{r}_{N}^{(N+1)}-\boldsymbol{r}_{0}^{(N)} \times d \boldsymbol{r}_{0}^{(N)} \\
= & M_{N+1} \boldsymbol{X}_{N+1} \times d \boldsymbol{X}_{N+1}+\frac{M_{N} m_{N+1}}{M_{N}+m_{N+1}}\left(\boldsymbol{x}_{N+1}-\boldsymbol{X}_{N}\right) \\
& \times\left(d \boldsymbol{x}_{N+1}-d \boldsymbol{X}_{N}\right)-M_{N} \boldsymbol{X}_{N} \times d \boldsymbol{X}_{N} \\
= & \frac{1}{M_{N+1}}\left(M_{N} \boldsymbol{X}_{N}+m_{N+1} \boldsymbol{x}_{N+1}\right) \times\left(M_{N} d \boldsymbol{X}_{N}+m_{N+1} d \boldsymbol{x}_{N+1}\right) \\
& +\frac{M_{N} m_{N+1}}{M_{N+1}}\left(\boldsymbol{x}_{N+1}-\boldsymbol{X}_{N}\right) \times\left(d \boldsymbol{x}_{N+1}-d \boldsymbol{X}_{N}\right)-M_{N} \boldsymbol{X}_{N} \times d \boldsymbol{X}_{N} \\
= & \frac{M_{N}^{2}+M_{N} m_{N+1}-M_{N} M_{N+1}}{M_{N+1}} \boldsymbol{X}_{N} \times d \boldsymbol{X}_{N}+\frac{m_{N+1}^{2}+M_{N} m_{N+1}}{M_{N+1}} \boldsymbol{x}_{N+1} \times d \boldsymbol{x}_{N+1} \\
= & m_{N+1} \boldsymbol{x}_{N+1} \times d \boldsymbol{x}_{N+1} .
\end{aligned}
$$

In the following, we fix the number of atoms $N$ and suppress the superscript $(N)$.

According to the relations (4.33) and (5.10), the inertia operator $\hat{I}_{x}: \mathbf{s o}(3) \rightarrow \mathbf{s o}(3) *$ is defined for each $\xi \in \mathbf{s o}(3) \cong \boldsymbol{R}^{3}$ by

$$
\hat{I}_{x}(\boldsymbol{\xi})=\hat{\boldsymbol{L}}\left(\theta_{x}(\boldsymbol{\xi})\right)=\sum_{i=0}^{N-1} \boldsymbol{r}_{i} \times\left(\boldsymbol{\xi} \times \boldsymbol{r}_{i}\right)=\sum_{i=0}^{N-1}\left(\left(\boldsymbol{r}_{i}, \boldsymbol{r}_{i}\right) \boldsymbol{\xi}-\left(\boldsymbol{\xi}, \boldsymbol{r}_{i}\right) \boldsymbol{r}_{i}\right)
$$

According as $x$ is a generic configuration, a planar one, a collinear one, or the collision one, the rank of $\hat{I}_{x}$ is $3,3,2$, or 0 , respectively. Unfortunately, there is no concise expression for the inverse $\left(\widetilde{I}_{x}\right)^{-1}$ in general. However, the connection form $\omega$ is expressed, from (4.36), as

$$
\omega=\widetilde{I}^{-1} \sum_{i=1}^{N-1} \boldsymbol{r}_{i} \times d \boldsymbol{r}_{i}
$$

To formulate molecular mechanics, we need the invariant volume form $v_{M}$ of $M$ associated with the metric $K$,

$$
v_{M}=d^{3} \boldsymbol{r}_{1} \wedge \cdots \wedge d^{3} \boldsymbol{r}_{N-1}
$$


Thus we have made a geometric setting to apply our formalism to molecular mechanics. However, before application we have to examine the topology of the orbit space (or shape space) $Q$ $=M / G$ for $N$-atomic molecules.

\section{B. Topology of the shape space}

Let $M(m, n)$ and $M(m, n)_{k}$ be the vector space of $m \times n$ matrices over $\boldsymbol{R}$, and the set of $m$ $\times n$ matrices of rank $k$, respectively. By $S(n)$ we denote the set of all the positive semi-definite symmetric $n \times n$ matrices, and set $S(n)_{k}:=S(n) \cap M(n, n)_{k}$. Of course, $k \leqslant m, n$. Let $\mathrm{O}(n)$ denote the orthogonal group acting on $\boldsymbol{R}^{n}$ as usual. Then $\mathrm{O}(m)$ and $\mathrm{O}(n)$ act on $M(m, n)$ to the left and to the right, respectively. We can verify now that

$$
M(m, n)_{k} \cong \frac{\mathrm{O}(m) / \mathrm{O}(m-k) \times S(k)_{k} \times \mathrm{O}(n-k) \backslash \mathrm{O}(n)}{\mathrm{O}(k)}
$$

from the observation of the fact that an arbitrary linear map $\varphi: \boldsymbol{R}^{n} \rightarrow \boldsymbol{R}^{m}$ of rank $k$ can be expressed as a composition $\varphi=i^{\circ} \sigma^{\circ} \pi$ of three linear maps, where $\pi, \sigma$, and $i$ are an orthogonal submersion $\pi: \boldsymbol{R}^{n} \rightarrow \boldsymbol{R}^{k}$, a positive-definite symmetric operator $\sigma: \boldsymbol{R}^{k} \rightarrow \boldsymbol{R}^{k}$, and an orthogonal immersion $i: \boldsymbol{R}^{k} \rightarrow \boldsymbol{R}^{m}$, respectively. Here we call a linear map $\pi: \boldsymbol{R}^{n} \rightarrow \boldsymbol{R}^{k}$ an orthogonal submersion, when it is surjective and satisfies $\pi^{\circ} \pi^{t}=\mathrm{id}$ on $\boldsymbol{R}^{k}$. Similarly, we call $i: \boldsymbol{R}^{k} \rightarrow \boldsymbol{R}^{m}$ an orthogonal immersion, when it is injective and satisfies ${ }^{t} i \circ i=\mathrm{id}$ on $\boldsymbol{R}^{k}$. The set of all the orthogonal submersions $\left\{\pi: \boldsymbol{R}^{n} \rightarrow \boldsymbol{R}^{k}\right\}$ is identified with a Stiefel manifold $\mathrm{O}(n-k) \backslash \mathrm{O}(n)$, while the set of all the orthogonal immersions $\left\{i: \boldsymbol{R}^{k} \rightarrow \boldsymbol{R}^{m}\right\}$ is identified with another Stiefel manifold $\mathrm{O}(m) / \mathrm{O}(m-k)$. An equivalence relation $\sim$ is defined on the triplet $(i, \sigma, \pi)$ by the action of $g \in \mathrm{O}(k)$ through $(i, \sigma, \pi) \sim\left(i g^{-1}, g \sigma g^{-1}, g \pi\right)$. In particular, for $m=n=k$, Eq. (5.16) becomes

$$
\mathrm{GL}(n)=M(n, n)_{n} \cong S(n)_{n} \times \mathrm{O}(n),
$$

which is nothing but the so-called polar decomposition of regular matrices.

Thus the configuration space of the molecule, $M=\left(\boldsymbol{R}^{3}\right)^{N-1}=M(3, N-1)$, is identified with

$$
M(3, N-1) \cong \cup_{0 \leqslant k \leqslant \min (3, N-1)} \frac{\mathrm{O}(3) / \mathrm{O}(3-k) \times S(k)_{k} \times \mathrm{O}(N-1-k) \backslash \mathrm{O}(N-1)}{\mathrm{O}(k)} .
$$

Each component with $k=0,1,2,3$ corresponds to the set of collision, collinear, planar, and generic configurations, respectively. Note that a point $x \in M$ is of the maximum type or of the singular type, according as $k=2,3$ or $k=0,1$. Strata of the shape space $Q=M / \mathrm{SO}(3)$ are then given by

$$
Q_{k}^{(N)} \cong \frac{\mathrm{SO}(3) \backslash \mathrm{O}(3) / \mathrm{O}(3-k) \times S(k)_{k} \times \mathrm{O}(N-1-k) \backslash \mathrm{O}(N-1)}{\mathrm{O}(k)} .
$$

The topology of strata for few-body problems with $N=3$ and $N=4$ is already studied by one of the authors ${ }^{7}$ and Narasimhan-Ramadas, ${ }^{28}$ respectively. Coordinates of the $N$-body problem are also studied by Littlejohn and Reinsch. ${ }^{29}$ They also wrote a comprehensive review ${ }^{30}$ on gauge fields in the $N$-body problem, and studied also complexes of rigid molecules. ${ }^{31}$

We write out topology with $N=2,3,4$ to give definite examples. We denote by $\boldsymbol{R}_{>0}$ the positive real numbers $(0, \infty)$ and by $D^{n}, S^{n}$, and $\boldsymbol{R} P^{n}$ an $n$-dimensional disk, sphere, projective space, respectively.

$$
N=2 \text { : }
$$

$$
\begin{gathered}
Q_{1}^{(2)} \cong S(1)_{1} \cong \boldsymbol{R}_{>0}, \\
Q_{0}^{(2)} \cong\{0\} .
\end{gathered}
$$

$N=3:$ 


$$
\begin{gathered}
Q_{2}^{(3)} \cong S(2)_{2} \cong \boldsymbol{R}_{>0} \times D^{2} \cong \boldsymbol{R}_{>0} \times \boldsymbol{R}^{2}, \\
Q_{1}^{(3)} \cong S(1)_{1} \times \frac{\mathrm{O}(2)}{\mathrm{O}(1) \times \mathrm{O}(1)} \cong S(2)_{1} \cong \boldsymbol{R}_{>0} \times \boldsymbol{R} P^{1} \cong \boldsymbol{R}_{>0} \times S^{1} \cong \boldsymbol{R}^{2}-\{0\}, \\
Q_{0}^{(3)} \cong\{0\} .
\end{gathered}
$$

$N=4$ :

$$
\begin{gathered}
Q_{3}^{(4)} \cong \frac{\mathrm{O}(3)}{\mathrm{SO}(3)} \times S(3)_{3} \cong \boldsymbol{Z}_{2} \times \boldsymbol{R}_{>0} \times D^{5} \cong \boldsymbol{R}_{>0} \times\left(S^{5}-S^{4}\right), \\
Q_{2}^{(4)} \cong \frac{S(2)_{2} \times \mathrm{O}(1) \backslash \mathrm{O}(3)}{\mathrm{O}(2)} \cong S(3)_{2} \cong \boldsymbol{R}_{>0} \times\left(S^{4}-\boldsymbol{R} P^{2}\right), \\
Q_{1}^{(4)} \cong \frac{S(1)_{1} \times \mathrm{O}(2) \backslash \mathrm{O}(3)}{\mathrm{O}(1)} \cong S(3)_{1} \cong \boldsymbol{R}_{>0} \times \boldsymbol{R} P^{2}, \\
Q_{0}^{(4)} \cong\{0\} .
\end{gathered}
$$

In the case of $N=3$, the union of $Q_{k}^{(3)}, k=0,1,2$, forms the shape space $Q \cong \boldsymbol{R}^{2} \times \boldsymbol{R}_{\geqslant 0}$. The maximum stratum is $Q_{2}^{(3)} \cong \boldsymbol{R}^{2} \times \boldsymbol{R}_{>0}$. For $N=4$, the union $Q_{3}^{(4)} \cup Q_{2}^{(4)}$ is the maximum stratum, which is diffeomorphic to $\boldsymbol{R}_{>0} \times\left(S^{5}-\boldsymbol{R} P^{2}\right)$.

\section{Triatomic molecules}

To make a practical application of the above general formalism, we concentrate on the triatomic molecules in the rest of the paper. The configuration space then becomes $M=\left(\boldsymbol{R}^{3}\right)^{2}$ $=\left\{\left(\boldsymbol{r}_{1}, \boldsymbol{r}_{2}\right)\right\}$. The maximum stratum $M_{\mu}$ is diffeomorphic with $M(3,2)_{2}$, the space of $3 \times 2$ matrices of maximal rank. The stratum of singular orbit type, $\partial M_{\mu}$, is the union $M(3,2)_{1} \cup M(3,2)_{0}$. Dragt $^{32}$ and his successors have introduced a useful coordinate system $(\alpha, \beta, \gamma, \rho, \chi, \phi)$ of $M$ by setting

$$
\begin{aligned}
& \boldsymbol{r}_{1}=\rho\left(\cos \frac{\chi}{2} \cos \frac{\phi}{2} \boldsymbol{u}_{3}+\sin \frac{\chi}{2} \sin \frac{\phi}{2} \boldsymbol{u}_{2}\right), \\
& \boldsymbol{r}_{2}=\rho\left(\cos \frac{\chi}{2} \sin \frac{\phi}{2} \boldsymbol{u}_{3}-\sin \frac{\chi}{2} \cos \frac{\phi}{2} \boldsymbol{u}_{2}\right) .
\end{aligned}
$$

Here $\left(\boldsymbol{u}_{1}, \boldsymbol{u}_{2}, \boldsymbol{u}_{3}\right)$ is an orthonormal basis of $\boldsymbol{R}^{3}$ parametrized by the Euler angles $(\alpha, \beta, \gamma)$ as

$$
\left(\boldsymbol{u}_{1}, \boldsymbol{u}_{2}, \boldsymbol{u}_{3}\right)=g\left(\boldsymbol{e}_{1}, \boldsymbol{e}_{2}, \boldsymbol{e}_{3}\right), \quad g=e^{\alpha J_{3}} e^{\beta J_{2}} e^{\gamma J_{3}},
$$

where $\left(\boldsymbol{e}_{1}, \boldsymbol{e}_{2}, \boldsymbol{e}_{3}\right)$ is the standard basis of $\boldsymbol{R}^{3}$ and $g$ is an element of $\mathrm{SO}(3)$ with $\left(J_{1}, J_{2}, J_{3}\right)$ the standard basis of so(3) defined by $J_{i} \boldsymbol{v}=\boldsymbol{e}_{i} \times \boldsymbol{v}(i=1,2,3)$ for each $\boldsymbol{v} \in \boldsymbol{R}^{3}$. We notice here that the orientation of the frame $\left(\boldsymbol{u}_{1}, \boldsymbol{u}_{2}, \boldsymbol{u}_{3}\right)$ is different from that of the original article. We choose the orientation to bring the collinear configurations into the direction of $\boldsymbol{u}_{3}$. The ranges of the coordinates are given by

$$
\begin{aligned}
& 0 \leqslant \alpha<2 \pi, \quad 0 \leqslant \beta \leqslant \pi, \quad 0 \leqslant \gamma \leqslant 2 \pi, \\
& 0 \leqslant \rho, \quad 0 \leqslant \chi \leqslant(\pi / 2), \quad 0 \leqslant \phi<2 \pi .
\end{aligned}
$$

The geometric meaning of $(\rho, \chi, \phi)$ is clear on introducing coordinates $\left(q_{1}, q_{2}, q_{3}\right)$ by 


$$
\begin{gathered}
q_{1}:=\left\|\boldsymbol{r}_{1}\right\|^{2}-\left\|\boldsymbol{r}_{2}\right\|^{2}=\rho^{2} \cos \chi \cos \phi, \\
q_{2}:=2\left(\boldsymbol{r}_{1}, \boldsymbol{r}_{2}\right)=\rho^{2} \cos \chi \sin \phi, \\
q_{3}:=2\left\|\boldsymbol{r}_{1} \times \boldsymbol{r}_{2}\right\|=\rho^{2} \sin \chi .
\end{gathered}
$$

They are invariant under the action of $\mathrm{SO}(3)$ on $M$, and provide the projection $\pi: M \rightarrow Q$ $=M / \mathrm{SO}(3) ;\left(\boldsymbol{r}_{1}, \boldsymbol{r}_{2}\right) \mapsto\left(q_{1}, q_{2}, q_{3}\right)$. With this expression of $\pi$, it is easy to show that $Q$ is homeomorphic to the upper half space $\boldsymbol{R}_{\geqslant 0}^{3}=\boldsymbol{R}^{2} \times \boldsymbol{R}_{\geqslant 0}$. The space, $\pi\left(M_{\mu}\right)$, of maximum orbits is diffeomorphic with $\boldsymbol{R}^{2} \times \boldsymbol{R}_{>0}$. The boundary surface, $\boldsymbol{R}^{2} \times\{0\}$, determined by $q_{3}=0$ or $\chi=0$ describes the orbit space for collinear configurations, in which the atoms make a line along $\boldsymbol{u}_{3}$. The origin $(0,0,0)$ represents the collision configuration. These observations coincide with (5.21). On the other hand, the Euler angles $(\alpha, \beta, \gamma)$ are regarded as a coordinate system of the fiber of the bundle $\pi: M_{\mu} \rightarrow Q_{\mu}$. Note that, in the set of singular points $\partial M_{\mu}$, one has $\boldsymbol{r}_{1}=\rho \cos (\phi / 2) \boldsymbol{u}_{3}$, $\boldsymbol{r}_{2}=\rho \sin (\phi / 2) \boldsymbol{u}_{3}$ with $\boldsymbol{u}_{3}=e^{\alpha J_{3}} e^{\beta J_{2}} \boldsymbol{e}_{3}$, which shows that $(\alpha, \beta)$ serve as coordinates for the orbit diffeomorphic with $S^{2}$, and that $(\rho, \phi)$ are coordinates for $\partial Q_{\mu} \cong \boldsymbol{R}^{2}$.

In terms of the coordinates introduced above, we are to write out the geometric objects in the explicit form. It is also convenient for later use to introduce the Maurer-Cartan one-form;

$$
\begin{aligned}
g^{-1} d g & =J_{1}(\sin \gamma d \beta-\sin \beta \cos \gamma d \alpha)+J_{2}(\cos \gamma d \beta+\sin \beta \sin \gamma d \alpha)+J_{3}(d \gamma+\cos \beta d \alpha) \\
& =J_{1} \Theta_{1}+J_{2} \Theta_{2}+J_{3} \Theta_{3} .
\end{aligned}
$$

Then the metric $g_{M}=K$ obtained in (5.9) takes the form,

$$
\begin{aligned}
g_{M}= & \left\|d \boldsymbol{r}_{1}\right\|^{2}+\left\|d \boldsymbol{r}_{2}\right\|^{2} \\
= & d \rho^{2}+\frac{1}{4} \rho^{2} d \chi^{2}+\frac{1}{4} \rho^{2} \cos ^{2} \chi d \phi^{2}+\rho^{2}\left(\Theta_{1}-\frac{1}{2} \sin \chi d \phi\right)^{2} \\
& +\rho^{2} \cos ^{2} \frac{\chi}{2} \Theta_{2}^{2}+\rho^{2} \sin ^{2} \frac{\chi}{2} \Theta_{3}^{2} .
\end{aligned}
$$

The angular momentum form $\hat{\boldsymbol{L}}: T M \rightarrow \mathbf{S o}(3)^{*} \cong \boldsymbol{R}^{3}$ obtained in (5.10) is expressed as

$$
\hat{\boldsymbol{L}}=\boldsymbol{r}_{1} \times d \boldsymbol{r}_{1}+\boldsymbol{r}_{2} \times d \boldsymbol{r}_{2}=\rho^{2} \boldsymbol{u}_{1}\left(\Theta_{1}-\frac{1}{2} \sin \chi d \phi\right)+\rho^{2} \cos ^{2} \frac{\chi}{2} \boldsymbol{u}_{2} \Theta_{2}+\rho^{2} \sin ^{2} \frac{\chi}{2} \boldsymbol{u}_{3} \Theta_{3}
$$

The inertia operator $\hat{I}_{x}: \mathbf{s o}(3) \cong \boldsymbol{R}^{3} \rightarrow \mathbf{s o}(3)^{*} \cong \boldsymbol{R}^{3}$ obtained in (5.13) is put in the form,

$$
\hat{I}_{x}(\boldsymbol{\xi})=\boldsymbol{r}_{1} \times\left(\boldsymbol{\xi} \times \boldsymbol{r}_{1}\right)+\boldsymbol{r}_{2} \times\left(\boldsymbol{\xi} \times \boldsymbol{r}_{2}\right)=\rho^{2}\left(\boldsymbol{\xi}, \boldsymbol{u}_{1}\right) \boldsymbol{u}_{1}+\rho^{2} \cos ^{2} \frac{\chi}{2}\left(\boldsymbol{\xi}, \boldsymbol{u}_{2}\right) \boldsymbol{u}_{2}+\rho^{2} \sin ^{2} \frac{\chi}{2}\left(\boldsymbol{\xi}, \boldsymbol{u}_{3}\right) \boldsymbol{u}_{3}
$$

The connection form $\boldsymbol{\omega}: T M \rightarrow \mathbf{S o}(3) \cong \boldsymbol{R}^{3}$ is then written out, according to (4.36), as

$$
\boldsymbol{\omega}=\widetilde{I}^{-1} \hat{\boldsymbol{L}}=\boldsymbol{u}_{1}\left(\Theta_{1}-\frac{1}{2} \sin \chi d \phi\right)+\boldsymbol{u}_{2} \Theta_{2}+\boldsymbol{u}_{3} \Theta_{3}
$$

Hence the vertical component of the metric (4.37) is given by

$$
g_{M} \mid V=I \circ(\boldsymbol{\omega} \otimes \boldsymbol{\omega})=(\hat{\boldsymbol{L}}, \boldsymbol{\omega})=\rho^{2}\left(\Theta_{1}-\frac{1}{2} \sin \chi d \phi\right)^{2}+\rho^{2} \cos ^{2} \frac{\chi}{2} \Theta_{2}^{2}+\rho^{2} \sin ^{2} \frac{\chi}{2} \Theta_{3}^{2}
$$

and thereby the metric $g_{Q}$ such that $\pi^{*} g_{Q}=g_{M} \mid H$ becomes

$$
g_{Q}=d \rho^{2}+\frac{1}{4} \rho^{2} d \chi^{2}+\frac{1}{4} \rho^{2} \cos ^{2} \chi d \phi^{2} .
$$


Then its inverse is a tensor field given by

$$
\left(g_{Q}\right)^{-1}=\frac{\partial}{\partial \rho} \otimes \frac{\partial}{\partial \rho}+\frac{4}{\rho^{2}} \frac{\partial}{\partial \chi} \otimes \frac{\partial}{\partial \chi}+\frac{4}{\rho^{2} \cos ^{2} \chi} \frac{\partial}{\partial \phi} \otimes \frac{\partial}{\partial \phi}
$$

The volume form defined by (5.31) is of the form

$$
v_{M}=\frac{1}{16} \rho^{5} \sin 2 \chi d \rho \wedge d \chi \wedge d \phi \wedge \Theta_{1} \wedge \Theta_{2} \wedge \Theta_{3} .
$$

Then the volume form associated with the measure (4.14) is

$$
v_{Q}=\frac{\pi^{2}}{2} \rho^{5} \sin 2 \chi d \rho \wedge d \chi \wedge d \phi
$$

since $\int \Theta_{1} \wedge \Theta_{2} \wedge \Theta_{3}=8 \pi^{2}$.

To describe quantum mechanics for the triatomic molecule, we need the Hilbert space of sections of vector bundles associated with the stratification. Any irreducible unitary representation of $\mathrm{SO}(3)$ is characterized by a nonnegative integer $l$ and denoted by $\rho^{l}: \mathrm{SO}(3) \rightarrow U\left(\boldsymbol{C}^{2 l+1}\right)$. We put $\hat{J}_{i}:=\left(\rho^{l}\right)_{*}\left(\boldsymbol{e}_{i}\right)$ for the standard basis $\boldsymbol{e}_{i} \in \boldsymbol{R}^{3} \cong \mathbf{S o}(3)$. Since the base space of the stratified fiber bundle $\pi: M \rightarrow Q \cong \boldsymbol{R}^{2} \times \boldsymbol{R}_{\geqslant 0}$ is contractible, the bundle is a trivial bundle. Through a global section $\sigma: Q \rightarrow M$ with $\alpha=\beta=\gamma=0$ in (5.23), (5.24), and (5.25), any equivariant function $\psi: M$ $\rightarrow C^{2 l+1}$ is pulled back to a function $\Psi:=\sigma^{*} \psi: \boldsymbol{R}_{\geqslant 0}^{3} \rightarrow \boldsymbol{C}^{2 l+1}$. Then the boundary condition (4.43) takes the form

$$
\hat{J}_{3} \Psi=0 \text { for } \chi=0
$$

on $M(3,2)_{1}$ and

$$
\hat{J}_{1} \Psi=\hat{J}_{2} \Psi=\hat{J}_{3} \Psi=0 \text { for } \rho=0
$$

on $M(3,2)_{0}$, respectively, with the coordinate system defined at (5.27), (5.28), and (5.29). What Eq. (5.40) means is as follows: At a collinear configuration determined by $\chi=0$, the molecule lying in the line along $\boldsymbol{u}_{3}$ has the vanishing angular momentum about $\boldsymbol{u}_{3} ;\left(\rho^{l}\right)_{*}\left(\boldsymbol{u}_{3}\right) \psi(x)=0$, so that one has $\rho^{l}(g)\left(\rho^{l}\right)_{*}\left(\boldsymbol{e}_{3}\right) \rho^{l}\left(g^{-1}\right) \psi(g \sigma(q))=0$ with $\boldsymbol{u}_{3}=g \boldsymbol{e}_{3}$, which is equivalent to (5.40). Since $\hat{J}_{3}=\operatorname{diag}(l, l-1, \ldots, 0, \ldots,-l)$, the components $\Psi_{m}$ of $\Psi$ with $m \neq 0$ vanish, if $l \neq 0$. Furthermore, Eq. (5.41) means that at the collision configuration determined by $\rho=0$, the molecule cannot carry nonzero angular momentum, so that the wave function can have a nonvanishing value only when $l=0$. These conditions are analogs of that for the two-dimensional case (4.47). Now we have implemented the consideration of singular case by providing the boundary condition (5.40) and (5.41) which we skipped in the previous work. ${ }^{7}$

In conclusion, we write down the Laplacian (4.42), combining (5.33), (5.34), (5.37), and (5.39),

$$
\begin{aligned}
-\Delta \Psi(\rho, \chi, \phi)= & \frac{1}{\rho^{5} \sin 2 \chi}\left\{\frac{\partial}{\partial \rho} \rho^{5} \sin 2 \chi \frac{\partial}{\partial \rho}+\frac{\partial}{\partial \chi} \frac{4}{\rho^{2}} \rho^{5} \sin 2 \chi \frac{\partial}{\partial \chi}\right. \\
& \left.+\left(\frac{\partial}{\partial \phi}+\frac{1}{2} \sin \chi \hat{J}_{1}\right) \frac{4}{\rho^{2} \cos ^{2} \chi} \rho^{5} \sin 2 \chi\left(\frac{\partial}{\partial \phi}+\frac{1}{2} \sin \chi \hat{J}_{1}\right)\right\} \Psi \\
& +\frac{1}{\rho^{2}}\left\{\left(\hat{J}_{1}\right)^{2}+\frac{1}{\cos ^{2}(\chi / 2)}\left(\hat{J}_{2}\right)^{2}+\frac{1}{\sin ^{2}(\chi / 2)}\left(\hat{J}_{3}\right)^{2}\right\} \Psi
\end{aligned}
$$




$$
\begin{aligned}
= & \left\{\frac{\partial^{2}}{\partial \rho^{2}}+\frac{5}{\rho} \frac{\partial}{\partial \rho}+\frac{4}{\rho^{2}}\left(\frac{\partial^{2}}{\partial \chi^{2}}+2 \cot 2 \chi \frac{\partial}{\partial \chi}\right)+\frac{4}{\rho^{2} \cos ^{2} \chi}\left(\frac{\partial}{\partial \phi}+\frac{1}{2} \sin \chi \hat{J}_{1}\right)^{2}\right\} \Psi \\
& +\frac{1}{\rho^{2}}\left\{\left(\hat{J}_{1}\right)^{2}+\frac{1}{\cos ^{2}(\chi / 2)}\left(\hat{J}_{2}\right)^{2}+\frac{1}{\sin ^{2}(\chi / 2)}\left(\hat{J}_{3}\right)^{2}\right\} \Psi
\end{aligned}
$$

which reproduces the result of Ref. 7. The first and last terms of the RHS of (5.42) are vibrational and rotational energy operators, up to sign, respectively. As was pointed out in Ref. 7, if the vibrational energy operator is separated off, and if the internal coordinates $(\rho, \chi, \phi)$ are fixed, the operator $\frac{1}{2} \Delta$ reduces to the well known Hamiltonian for a rigid rotor of plane body.

\section{CONCLUDING REMARKS}

In this paper we formulated the general method of reduction of quantum systems with symmetry by the use of the Peter-Weyl theorem. Although the method is well-known implicitly among Physicists, we developed it explicitly to give rigorous grounds to quantum mechanics describing molecular motions. We studied the stratification of manifolds according to the action of a symmetry Lie group and then defined a stratified bundle and a stratified connection as generalization of fiber bundles and connections. Further, we showed that the reduced quantum system is a pair of the Hilbert space and the Hamiltonian which are the space of equivariant functions and the Laplacian expressed in terms of covariant derivation with the stratified connection, respectively. We found the boundary condition that is imposed on the equivariant functions to make the reduced Laplacian a self-adjoint operator. Finally, the general formulation for reduction was applied to $\mathrm{N}$-atomic molecules, and triatomic molecules were examined in particular.

The stratified connection on the stratified bundle is newly introduced as a generalization of connections on principal fiber bundles and is used to describe the reduced Laplacian. One of our main results is to have determined the boundary condition for making the reduced Laplacian self-adjoint. Emmrich and H. Römer ${ }^{33}$ analyzed Laplacians on orbifolds to study quantization of systems with gauge symmetry. They found that the Laplacian on an orbifold is not essentially self-adjoint and therefore its self-adjoint extension is not unique. According to our method, the reduced Laplacian is self-adjoint by its definition and the boundary condition is accompanied automatically by the symmetry.

We would like to make some comments on remaining problems. First, although we built a general formulation to deal with quantum molecular systems, we do not obtain spectrum of the reduced Hamiltonian. Even the three-body problem is difficult to solve. It is desired to develop an approximate method to solve the reduced eigenvalue problem of physically interesting systems.

Second, for application to real molecules, electronic structure must be considered. Of course, spins and statistics of electrons and nucleus also must be taken into account. These are left for future investigation.

\section{ACKNOWLEDGMENTS}

The authors would like to thank Professor Uwano for helpful discussion. They are also grateful to Professor Tachibana for bringing our attention to problems of molecular quantum mechanics. This work was supported by a Grant-in-Aid for Scientific Research from the Ministry of Education, Science, and Culture of Japan.

\footnotetext{
${ }^{1}$ J. Marsden and A. Weinsten, Rep. Math. Phys. 5, 121 (1974).

${ }^{2}$ R. Abraham and J. E. Marsden, Foundations of Mechanics, 2nd ed. (Benjamin/Cummings, Reading, MA, 1978).

${ }^{3}$ A, Guichardet, Ann. Inst. Henri Poincare 40, 329 (1984).

${ }^{4}$ T. Iwai, Ann. Inst. Henri Poincare 47, 199 (1987).

${ }^{5}$ T. Iwai, J. Phys. A 31, 3849 (1998).

${ }^{6}$ T. Iwai, J. Math. Phys. 28, 964 (1987).

${ }^{7}$ T. Iwai, J. Math. Phys. 28, 1315 (1987).

${ }^{8}$ A. Tachibana and T. Iwai, Phys. Rev. A 33, 2262 (1986).
} 
${ }^{9}$ T. Iwai, J. Math. Phys. 40, 2381 (1999).

${ }^{10}$ M. Kummer, Indiana Univ. Math. J. 30, 281 (1981).

${ }^{11}$ W. J. Satzer, Jr., Indiana Univ. Math. J. 26, 951 (1977).

${ }^{12}$ P. A. M. Dirac, The Principles of Quantum Mechanics, 4th ed. (Oxford University Press, Oxford, 1958).

${ }^{13}$ P. A. M. Dirac, Proc. R. Soc. London, Ser. A 246, 333 (1958).

${ }^{14}$ V. Moncrief, Phys. Rev. D 5, 277 (1972).

${ }^{15}$ N. P. Landsman and N. Linden, Nucl. Phys. B 365, 121 (1991).

${ }^{16}$ N. P. Landsman, J. Geom. Phys. 15, 285 (1995).

${ }^{17}$ K. K. Wren, J. Geom. Phys. 24, 173 (1998).

${ }^{18}$ M. A. Rieffel, Adv. Math. 13, 176 (1974).

${ }^{19}$ S. Tanimura and I. Tsutsui, Mod. Phys. Lett. A 10, 2607 (1995).

${ }^{20}$ S. Tanimura and I. Tsutsui, Ann. Phys. 258, 137 (1997).

${ }^{21}$ J. Śniatycki and A. Weinstein, Lett. Math. Phys. 7, 155 (1983).

${ }^{22}$ J. M. Arms, M. J. Gotay, and G. Jennings, Adv. Math. 79, 43 (1990).

${ }^{23}$ M. Davis, Pac. J. Math. 77, 315 (1978).

${ }^{24}$ M. Davis, Multiaxial Actions on Manifolds, Lecture Notes in Mathematics 643 (Springer, Berlin, 1978).

${ }^{25} \mathrm{G}$. E. Bredon, Introduction to Compact Transformation Groups (Academic, New York, 1972).

${ }^{26}$ J. M. Souriau, Structure des Systèmes Dynamiques (Dunod, Paris, 1975).

${ }^{27}$ J. C. Simo, D. R. Lewis, and J. E. Marsden, Arch. Ration. Mech. Anal. 115, 15 (1991).

${ }^{28}$ M. S. Narasimhan and T. R. Ramadas, Commun. Math. Phys. 67, 121 (1979).

${ }^{29}$ R. G. Littlejohn and M. Reinsch, Phys. Rev. A 52, 2035 (1995).

${ }^{30}$ R. G. Littlejohn and M. Reinsch, Rev. Mod. Phys. 69, 213 (1997).

${ }^{31}$ K. A. Mitchell and R. G. Littlejohn, Mol. Phys. 96, 1305 (1999).

${ }^{32}$ A.J. Dragt, J. Math. Phys. 6, 533 (1965).

${ }^{33}$ C. Emmrich and H. Römer, Commun. Math. Phys. 129, 69 (1990). 